\title{
PGRMC1 effects on metabolism, genomic mutation and CpG methylation imply crucial roles in animal biology and disease
}

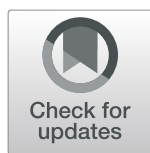

\author{
Bashar M. Thejer 1,2, Partho P. Adhikary ${ }^{1,3}$, Sarah L. Teakel', Johnny Fang1, Paul A. Weston ${ }^{4,5}$, Saliya Gurusinghe 4 \\ Ayad G. Anwer ${ }^{6,7}$, Martin Gosnell ${ }^{6,8}$, Jalal A. Jazayeri ${ }^{1}$, Marina Ludescher ${ }^{9}$, Lesley-Ann Gray ${ }^{10}$, Michael Pawlak ${ }^{11}$, \\ Robyn H. Wallace ${ }^{1}$, Sameer D. Pant ${ }^{12}$, Marie Wong ${ }^{13}$, Tamas Fischer ${ }^{14}$, Elizabeth J. New ${ }^{15}$, Tanja N. Fehm ${ }^{9}$, \\ Hans Neubauer ${ }^{9}$, Ewa M. Goldys ${ }^{6,7}$, Jane C. Quinn ${ }^{4,16}$, Leslie A. Weston ${ }^{4,5}$ and Michael A. Cahill ${ }^{1,14^{*}}$
}

\begin{abstract}
Background: Progesterone receptor membrane component 1 (PGRMC1) is often elevated in cancers, and exists in alternative states of phosphorylation. A motif centered on PGRMC1 Y180 was evolutionarily acquired concurrently with the embryological gastrulation organizer that orchestrates vertebrate tissue differentiation.

Results: Here, we show that mutagenic manipulation of PGRMC1 phosphorylation alters cell metabolism, genomic stability, and CpG methylation. Each of several mutants elicited distinct patterns of genomic CpG methylation. Mutation of S57AY180/S181A led to increased net hypermethylation, reminiscent of embryonic stem cells. Pathways enrichment analysis suggested modulation of processes related to animal cell differentiation status and tissue identity, as well as cell cycle control and ATM/ATR DNA damage repair regulation. We detected different genomic mutation rates in culture.

Conclusions: A companion manuscript shows that these cell states dramatically affect protein abundances, cell and mitochondrial morphology, and glycolytic metabolism. We propose that PGRMC1 phosphorylation status modulates cellular plasticity mechanisms relevant to early embryological tissue differentiation.
\end{abstract}

Keywords: Epigenetics, Genomic sequence, Hyperspectral autofluorescence, Organizer, Embryology, Metabolism, Cell death, Cytochrome P450, Steroid biology

\section{Summary statement}

PGRMC1 phosphorylation site mutations cause pronounced metabolic changes, genomic mutation rates, and altered genomic CpG methylation, without affecting progesteronedependent doxycycline resistance. Hypermethylation of a S57A/Y180F/S181A mutant resembles pluripotent stem cells.

\footnotetext{
* Correspondence: mcahill@csu.edu.au

'School of Biomedical Sciences, Charles Sturt University, Wagga Wagga, NSW 2650, Australia

${ }^{14}$ ACRF Department of Cancer Biology and Therapeutics, The John Curtin School of Medical Research, The Australian National University, Canberra, ACT 2601, Australia

Full list of author information is available at the end of the article
}

\section{Background}

Progesterone (P4) receptor membrane component 1 (PGRMC1) is a cytochrome $b_{5}$ (cytb5) protein and the archetypal a member of the membrane-associated P4 receptor (MAPR) family. It has a plethora of reported functions [1-4]. Human PGRMC1 contains predicted binding site motifs for Src homology 2 (SH2) and Src homology 3 (SH3) domain-containing proteins, with several other phosphorylation sites at S57, T178 and S181 being thought to additionally regulate these sites [5]. The $\mathrm{SH} 3$ target motif adjacent to S57, and the $\mathrm{SH} 2$ target motifs containing Y139 and T178/Y180/S181 are mutually juxtaposed on the folded protein surface, forming a potential proximity-stimulated

(c) The Author(s). 2020 Open Access This article is licensed under a Creative Commons Attribution 4.0 International License, which permits use, sharing, adaptation, distribution and reproduction in any medium or format, as long as you give appropriate credit to the original author(s) and the source, provide a link to the Creative Commons licence, and indicate if changes were made. The images or other third party material in this article are included in the article's Creative Commons licence, unless indicated otherwise in a credit line to the material. If material is not included in the article's Creative Commons licence and your intended use is not permitted by statutory regulation or exceeds the permitted use, you will need to obtain permission directly from the copyright holder. To view a copy of this licence, visit http://creativecommons.org/licenses/by/4.0/ The Creative Commons Public Domain Dedication waiver (http://creativecommons.org/publicdomain/zero/1.0/) applies to the data made available in this article, unless otherwise stated in a credit line to the data. 
tripartite signaling platform $[5,6]$. Thus, PGRMC1 represents a potential integration point and effector of many cell signaling pathways responsible for growth and proliferation $[2,3,6,7]$.

The PI3K/Akt pathway has been shown to be affected by over expression of PGRMC1 [8], or 3x hemagglutinin (HA)-tagged PGRMC1 (PGRMC1-HA) phosphorylation site mutants in MCF-7 cells [9]. A PGRMC1-HA S57A/ S181A double mutant (DM) was associated with differential cell survival to oxidative stress. Furthermore, PGRMC1 was shown to be differentially phosphorylated between estrogen receptor-positive and negative breast cancers [9]. This study aims to further explore the biology of these PGRMC1 phosphorylation sites.

In a companion publication designed to investigate the biology of the DM mutation [10], we found that stably expressed DM PGRMC1-HA induced dramatic effects in MIA PaCa-2 (MP) pancreatic cancer cells relative to cells expressing wild-type PGRMC1-HA (WT), including elevated PI3K/Akt activity, altered metabolism, mitochondrial morphology and function, actin cytoskeleton proteins, and elevated migration rates relative to WT cells. To test the hypothesis that the DM effects required Y180, further mutation of S57A/Y180F/S181A generated triple mutant (TM) cells (which differ to DM cells by just the phosphate acceptor of Y180). Importantly, S57 and Y180/S181 (mutated in this study) probably occupy conformationally flexible regions in solution, immediately $\mathrm{N}$ - and C-terminally flanking the cytb5/MAPR domain. These are hence adjacent to each other in the folded protein structure $[5,11]$, such that these mutations are unlikely to induce large conformational changes in the folded cytb5/MAPR domain. Indeed, these residues all appeared outside the MAPR domain during evolution of the animal lineage leading to humans [12].

TM cells exhibited reversal of some DM effects, including lowering of PI3K/Akt and migration activity, indicating that the DM phenotype was reliant upon Y180, and also led to dramatically impaired mouse xenograft tumor formation. Pathways enrichment analysis of a proteomics study suggested broad effects that were suggestive of plastic cellular reprogramming [10]. In this study we were interested in exploring which known or newly discovered PGRMC1 functions are related to these phosphorylation events. In this context it is informative to consider when different PGRMC1 functions may have been acquired during evolution because evolutionary conservation is a strong indicator of functional importance.

PGRMC1 functions include some cytb5-typical functions that predate or are potentially ancient, by which we mean functions that were inherited into animals from single celled eukaryotes such as regulation of heme synthesis [13], cytochrome P450 (cyP450) interactions [1, 14, 15], and sterol metabolism [16-18]. Some functions evidently arose in eukaryotes (e.g. membrane trafficking (reviewed by: $[2,19])$, cell cycle regulation at the G0/G1 checkpoint $[2,20,21]$, mitotic/meiotic spindle association [22-24]). Other clearly specialized metazoan functions include e.g., hormonal influence on fertility, embryogenic axon guidance, and membrane trafficking associated with synaptic plasticity [reviewed by: 2, 25]. The PGRMC1 protein is accordingly found in diverse subcellular locations, including endoplasmic reticulum/perinuclear membrane and Golgi apparatus [9, 25], at the cell surface [26, 27], mitochondria [13], and the nucleus, where it localizes to nucleoli [28].

PGRMC1 interacts with and regulates ferrochelatase, the mitochondrial rate-limiting enzyme in heme synthesis [13]. The related protein PGRMC2 has recently been reported to chaperone heme from mitochondria to the nucleus in adipocytes, where heme regulates the transcription of genes that encode mitochondrial proteins. Lack of PGRMC2 led to altered mitochondrial morphology and function [29], superficially reminiscent of the mitochondrial effects of PGRMC1 phosphorylation mutants in MIA PaCa-2 cells [10].

PGRMC1 is induced by a variety of agents causing DNA damage, including the topoisomerase II inhibitor doxorubicin [30]. The PGRMC1 yeast Dap1 (damage-associated protein 1) homologs are associated with DNA damage response $[17,31]$. Heme-binding and cyP450 interactions are thought to be required for DNA damage response [17, 30], which seems to be an ancient function.

PGRMC1 is strongly associated with sterol biology including association with the Insig/SCAP/SREBP complex that senses cholesterol levels and leads to mevalonate pathway induction [32] and production of the first sterol, lanosterol (reviewed by: $[6,16]$ ), which are apparently ancient functions. CyP450-interactions [1] conspicuously include PGRMC1 regulation of the most conserved eukaryotic cyP450 (lanosterol 14-alpha demethylase, CYP51A) to modify lanosterol from yeast to mammals which leads to cholesterol synthesis, providing the substrate for subsequent vertebrate steroidogenesis $[6,17,18]$. The PGRMC1mediated conferral of responsiveness to steroids like P4 presumably evolved in animals [33]. A complex between PGRMC1, the low density lipoprotein (LDL) receptor (LDLR) and the sigma-2 receptor transmembrane protein 97 (TMEM97) regulates the rapid internalization of LDLR via the membrane trafficking function of PGRMC1 [34], which is clearly an animal invention related to intercellular lipid transport, but related to ancient PGRMC1 steroid biology functions [16].

Anti-apoptotic activity of PGRMC1, especially to treatment with doxorubicin, has been reported by several studies and is linked to PGRMC1-dependent P4 responsiveness [35-37]. In mammals it involves PGRMC1 association with the CYP2D6 and CYP3A4 cyP450 enzymes responsible for doxorubicin hydroxylation and inactivation, and this activity required the PGRMC1 heme-chelating residue Y113 
[11]. However, it remains unclear whether heme-chelation or tyrosine phosphorylation (and perhaps membrane trafficking) of Y113 is involved [5]. Therefore PGRMC1dependent P4-responsiveness may involve the membrane trafficking of actual $\mathrm{P} 4$ receptors to the cell surface, or PGRMC1 itself may mediate its own P4 response (reviewed by $[2,30])$. PGRMC1 undeniably confers P4 responsiveness to cells of the reproductive [3] and the central nervous systems [4]. A D120G heme binding-incapacitated mutant exhibited reduced P4-responsiveness [37], implicating hemebinding requirement for the $\mathrm{P} 4$ response. While steroid hormone signaling appeared with eumetazoans [33], apoptosis is employed widely by animals but it is widespread in unicellular and multicellular eukaryotes. Some underlying mechanisms were indeed inherited from protomitochondrial bacterial contributions to eukaryogenesis [38]. So it is difficult to judge whether anti-apoptosis is an ancient or an animal phenomenon.

To assay function, pharmacologists preferentially employ specific inhibitors. The AG-205 small molecule inhibitor of PGRMC1 is frequently used to study the role of PGRMC1 in various cellular processes. We have recently shown that AG-205 treatment results in the failure of many proteins associated with the actin cytoskeleton to immunoprecipitate with PGRMC1 [39]. Note that AG205 is often cited as a PGRMC1-specific inhibitor. While it certainly affects PGRMC1-dependent function, AG-205 was modelled in silico against the heme-binding site of a plant MAPR protein [40]. As noted previously [2], there is no evidence that it is any more specific to PGRMC1 than to PGRMC2, or other MAPR family members Neudesin or Neuferricin, or indeed that it does not exert effects involving non MAPR proteins. Claims related to "PGRMC1specific inhibitor AG-205" should be treated circumspectly. Notwithstanding, we address AG-205 responsiveness of PGRMC1 mutations here, which are likely to reflect ancient MAPR biology.

In summary of the above, PGRMC1 has been reported to be involved in a plethora of different functions, potentially important to several central aspects of eukaryotic biology, and which seem to have evolved at different times. At least some of these seem to be regulated by phosphorylation events that appeared in eumetazoan animals [12], whereas other functions seem to be ancient in eukaryotes. Almost nothing is known about many functions. It remains unclear which functions are inhibited by the inhibitor AG205, and how these are related to the PGRMC1-specific P4 response. There is an urgent need to systematically stratify and separate PGRMC1 functions. Here, we sought to further characterize the cells described in the companion paper [10] in an effort to better understand the biology. We report no detectable influence on P4- or AG-205 responses, but we observed pronounced effects on metabolism, genomic stability and epigenetic plasticity, implicating the PGRMC1 Y180 module with critical newly identified eumetazoan roles.

\section{Results \\ PGRMC1 phosphorylation state-dependent metabolic differences}

In order to assess metabolic differences, cells were subjected to preliminary hyperspectral autofluorescence imaging: a non-invasive analytical technique [41]. Distinct spectral signatures of endogenous auto-fluorescent molecules $[42,43]$ can provide valuable information about intracellular metabolic state [44]. In a pilot study we showed that MP and DM cells exhibited detectably different metabolites [43]. As a first step towards demonstrating altered PGRMC1-dependent metabolic processes, eight informative spectral features that discriminated the cells were chosen (Fig. 1a and Table S1). The cells in each condition could be clearly distinguished using the autofluorescence of their endogenous metabolites. A pairwise linear discrimination analysis using the same spectral features indicated that all cell conditions were highly significantly separated from each other on the basis of endogenous autofluorescence (Fig. 1a).

Figure $1 \mathrm{~b}$ shows autofluorescence differences in spectral channel 3, thought to reflect flavin emission [44, 45]. All cells over-expressing PGRMC1-HA proteins exhibited reduced levels, with DM and TM the lowest (Fig. 1b). We also noted significant differences observed between cell lines for autofluorescent emission at $700 \mathrm{~nm}$ (Fig. S1A), in a wavelength range at which heme-containing proteins and other porphyrins contribute strongly to autofluorescence [46]. Note that PGRMC1 not only binds heme but is involved in heme synthesis [13]. Heme itself does not emit fluorescence but leads to fluorescence quenching, however, various heme-containing proteins are fluorescent [46]. Although the actual identities of these and fluorescent species contributing to many hyperspectral channels remain unknown, several unidentified parameters also significantly discriminated between the metabolites present in the different cell lines, such as e.g. the ratio of channel 3 to channel 12 (Fig. S1B,C, Table S1). These results indicated that PGRMC1 phosphorylation status dramatically alters cell metabolic state. In particular, the difference between DM and TM cells is due to the oxygen acceptor atom of Y180.

\section{PGRMC1 phosphorylation mutants did not affect P4 or AG-205 responses}

We were interested in whether DNA mutation rates may have been related to the P4-dependent protection against cell death, or to the mechanism of AG-205-induced cell death. Consistent with previously reported PGRMC1dependent anti-mitotic effects of P4 in Ishikawa endometrial cancer cells [36] and MDA-MB-231 breast cancer cells [47], incubation of cells in $1 \mu \mathrm{M}$ P4 retarded cell proliferation of 

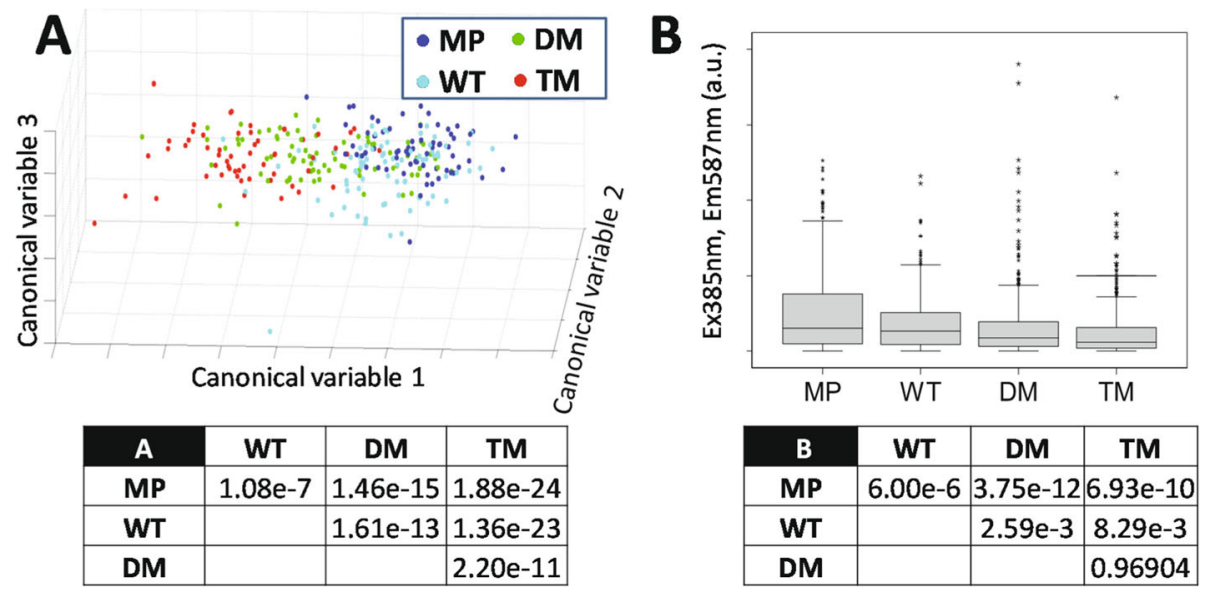

Fig. 1 Hyperspectral autofluorescence reveals metabolic differences between cells expressing different mutant PGRMC1-HA proteins. a Principal component analysis (PCA) followed by linear discriminant analysis (LDA) reveal hyperspectral autofluorescence parameters that significantly discriminate between cell types. The panel provides a three dimensional depiction of the three most significant canonical variables revealed by PCA and LDA as described [44] that differ according to PGRMC1-HA phosphorylation status. The table provides $p$ values from KolmogorovSmirnov test pairwise discrimination analysis. b Mean cellular intensity of hyperspectral autofluorescence channel 3 [375 nm(Ex), $450 \mathrm{~nm}(\mathrm{Em})]$, which corresponds primarily to flavin emission, is significantly affected by PGRMC1-HA phosphorylation status. Boxplots were generated in SPSS. The table shows Kruskal-Wallis test $p$ values for the pair-wise condition comparisons performed on primary emission data

all cells over-expressing a PGRMC1-HA protein (WT, DM or TM), but not of MP cells relative to non-P4-treated control cells (Fig. 2a). When cells pretreated with or without P4 for $1 \mathrm{~h}$ were co-incubated in the presence doxorubicin (Dox), in the absence of P4 then WT, DM and TM cells were more susceptible to Dox-induced death (Fig. 2b-c, white data points and boxes), however these cells exhibited greater P4-dependent protection against Dox-induced death (Fig. 2b-c, shaded data points and boxes). As reported previously for Ishikawa cells [36], the $1 \mathrm{~h}$ pre-incubation with P4 was required to observe these protective effects of P4 (data not shown). There was no significant protective effect of P4
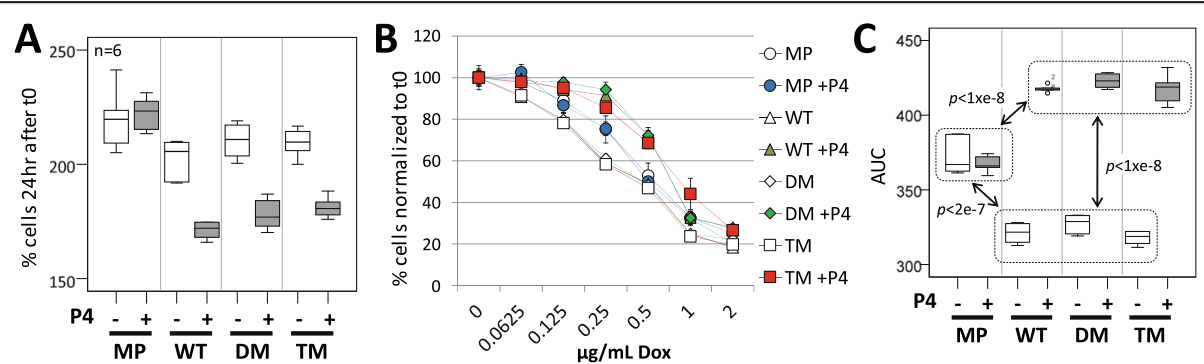

Fig. 2 PGRMC1 phosphorylation status does not affect P4-dependent resistance to doxorubicin toxicity or resistance to AG-205-induced cell death. a P4 reduces cell proliferation of cells expressing all PGRMC1-HA proteins. The panel shows boxplots of viable cells for $n=6$ replicates. The viability of cells pretreated with P4 (dark boxes) or DMSO vehicle control (light boxes) after $23 \mathrm{~h}$ were allowed to grow a further $24 \mathrm{~h}$ and the level of MTT formazan was quantified as a proxy for viable cell numbers. "\% cells after $48 \mathrm{hr}$ " is presented relative to the signal obtained after adherence for $3 \mathrm{~h}$. No significant differences were observed between non-P4 treated cell pairings, except MP v. TM ( $p=0.021,2$ way ANOVA). Pair wise comparisons of P4-treated MP vs any of P4-treated WT, DM or TM, or of +/-P4 treatment for WT, DM and TM, were significantly different at the $p<0.00001$ level (2 way ANOVA). MP cells $+/-\mathrm{P} 4$ were not different $(p=0.55$, two way ANOVA). Considering only P4-treated cells, MP differed in P4 response from all other cell types $\left(p<1 \times 10^{-10}\right)$, and WT differed marginally from TM $(p=0.034)$ by one way ANOVA and post-hoc Bonferroni. b P4-protection of MP cells from doxorubicin-induced cell death is facilitated by over-expression of PGRMC1-HA proteins (WT, DM \& TM). Cells were grown as in (a), except at t0 doxorubicin (Dox) was added at the indicated concentrations, followed by $24 \mathrm{~h}$ incubation. Because of altered cell proliferation during pretreatment with P4 (a), all signals at t0 were expressed as a percentage of the averaged control sample without Dox at to to assess the effects of P4. Data points represent the averages \pm s.d. of $n=6$ replicates. $\mathbf{c}$ Boxplots of area under the curve (AUC) results for all data points with dox addition from (b). Two way ANOVA dox treatments were statistically significant $(F=1292.237, \mathrm{df}=1$, $\left.\mathrm{df} 2=40, p<1=\mathrm{e}^{-8}\right)$, with Partial Eta Squared indicating $97 \%$ effect size in the data. Considering pairwise comparisons of cell classes, DM v. TM $(p=0.014)$ and WT v. DM $(p=0.05)$ were significantly different. Pairwise comparison between the AUC levels for $+/-\mathrm{P} 4$ for each cell class yielded no difference for MP, but $p<1 \times 10^{-8}$ for other cell types, $+/-$ P4. One way ANOVA post-hoc Bonferroni pairwise comparisons for $-\mathrm{P} 4$ cells revealed that samples within dotted boxes did not differ significantly, whereas all pairwise $p$ values were less than those indicated for comparisons between boxes 
on cell survival for MP cells (Fig. 2c). Notably, there were no significant differences in P4-dependent protection between WT, MP or TM cells. There was also no difference in the sensitivity of any of these cells to AG-205-induced cell death, with essentially superimposable dose response curves, and a half maximal lethal effective concentration $\left(\mathrm{EC}_{50}\right)$ of approximately $32 \mu \mathrm{M}$ AG-205 (Fig. S2). Therefore, although we observe PGRMC1-HA-dependent response to $\mathrm{P} 4$, this response is apparently unaffected by the influence of our PGRMC1 phosphorylation mutants. We conclude that the dramatic effects of altered PGRMC1 phosphorylation status observed in this and the accompanying manuscript [10] are due to different and newly described functions of PGRMC1, unrelated to the mechanism of P4induced vitality or AG-205-induced death. However, our mutant effects may require processes that emanate from the heme-binding MAPR domain, since the wild-type MAPR domain sequence was present in all mutants.

\section{PGRMC1 phosphorylation status affects cytoplasmic redox status}

Changes in metabolism could be associated with altered states of oxidative environment. Naphthalimide-flavin redox sensor 1 (NpFR1) is a fluorophore analogous to mitochondrial-specific NpFR2 [10, 48], except that it is localized to the cytoplasm [49]. To further explore metabolic state, cells were treated with NpFR1 and fluorescent state was assayed by flow cytometry to reveal two cell populations: one with less oxidized NpFR1 and one with more oxidized NpFR1 (Fig. 3a). The type of experiment shown in Fig. 3 has been performed on numerous occasions. The actual ratio of low to high abundance distribution for a cell type tends to vary between experiments on different days, however the inter-cell comparisons of cells in the low fluorescent population between cell types examined on a single day remains relatively constant $(\mathrm{MP}>\mathrm{DM}>\mathrm{WT}>\mathrm{TM})$.

The bimodal fluorescent peaks of Fig. 3a represent cell populations with different levels of cytoplasmic oxidation/reduction which have not been further characterized. All PGRMC1-HA-expressing cells exhibited the majority of cells in a more oxidized peak. TM (9.4\%) and WT (17.7\%) cells both exhibited less cells in the lower oxidized fraction (Fig. 3a,b, black arrows). For TM cells, which had most cells in the more oxidizing cytoplasmic population, the degree of NpFR1 oxidation in that fraction was significantly lower than for all other cells (Fig. 3a,c, white arrow). Higher cytoplasmic oxidative levels in TM cells suggested potential for effects on genomic mutation rates, associated with Y180 function.

\section{PGRMC1 phosphorylation status affects pathways associated with replication, cell cycle, and mitogenic signaling}

In the accompanying publication [10] we presented only highly significantly differential WebGestalt proteomics pathways with Benjamini-Hochberg adjusted $p$ (adjP) values $<0.001$. Several pathways related to cell cycle control and DNA replication were also detected, albeit with weaker significance, including five proteins predicted to be associated with decreased levels of ATM/ATR DNA damage sensing and control in TM cells (Fig. 4a), which was interesting to us because of the DNA damage associations of PGRMC1 (see Background). Although we could not detect differences in cultured proliferation of any cell lines [10], reverse phase protein array (RPPA) analysis revealed elevated levels of activated MKK4, MEK and ERK, as well as phosphorylated retinoblastoma protein $(\mathrm{Rb})$ in TM cells (Fig. 4b). This suggests that PGRMC1 Y180 may be somehow involved in regulating the G1 checkpoint, which may be related to the reported ability of PGRMC1 and/or PGRMC1 to lower the propensity of cells to enter the G1 and the cell cycle, rather than enter G0, in spontaneously immortalized granulosa cells [20]. However, this hypothesis requires further investigation.

PGRMC1 phosphorylation status affects genomic integrity Altered cytoplasmic oxidative environment, potential perturbations of the ATM/ATR pathways, and possible G1 checkpoint features without detectable differences in proliferation rates [10] led us to explore genomic stability. We cultured one cell line each of MP, WT, DM and TM for 30 passages, and sequenced the genomes of each. The PGRMC1-HA constructs were expressed at similar levels after 30 passages (Fig. 5a). Relative to the reference human genome sequence, the order of average mutation rates (difference to reference) per chromosome were $\mathrm{WT}<\mathrm{DM}<\mathrm{TM}$ (not shown), which was also reflected in unique mutations per $\mathrm{MB}$, as rates per chromosome (Fig. 5b). This indicates that manipulation of the phosphorylation status of PGRMC1 Y180 can affect genomic stability, which is of potentially considerable relevance to cancer biology.

\section{PGRMC1 phosphorylation status regulates the NNMT/1- MNA pathway}

To further characterize metabolic differences between cells (Fig. 1) we performed a pilot metabolomics study, where we observed elevated levels of 1-methylnicotinamide (1-MNA) in some but not all experiments (not shown), which we nevertheless chose to explore. 1-MNA is produced by the enzyme nicotinamide- $\mathrm{N}$-methyl transferase (NNMT), which we found to be elevated in TM cells (Fig. 6a). NNMT utilizes S-adenosylmethionine (SAM) as a methyl group donor to convert nicotinamide to 1-MNA [50] (Fig. 6b).

Liquid chromatography/mass spectrometry (LC/MS) quantification revealed lower levels of SAM in DM and TM cells (Fig. S4A). Anti-NNMT shRNA attenuated NNMT levels in TM cells more than two-fold (Fig. S4B), which led to expected reduced levels of 1-MNA (Fig. S4C). 


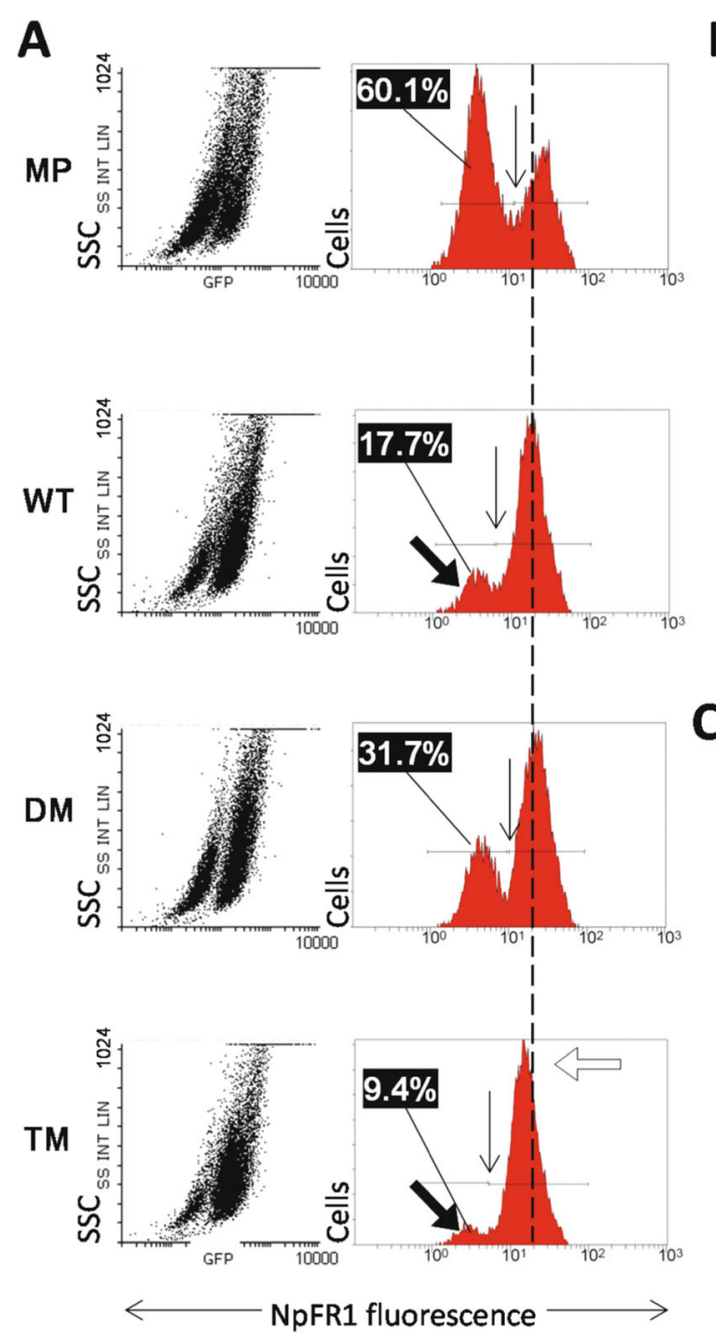

B
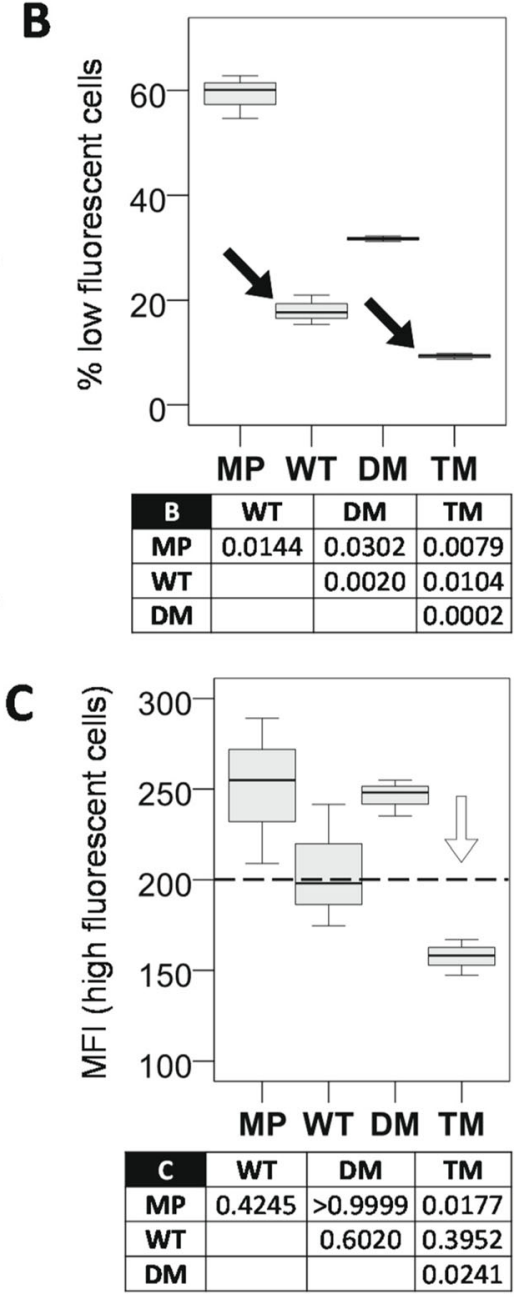

Fig. $3 \mathrm{NpFR} 1$ reveals differences in cytoplasmic redox status. a NpFR1 flow cytometry results. Scatter plots to the left depict green fluorescence on the $x$ axis, and side scatter on the $y$ axis. In the right panels the $y$ axis represents cell number. The numbers at the upper left of each right panel are the fraction of the population in the respective left hand low fluorescent peak. Vertical arrows show the boundary between cell populations, and the inset numbers in the left of each panel represent the percentage of cells in the less oxidized left hand cell population. The vertical dotted reference line represents the 200 fluorescence intensity units, and the open arrow highlights TM fluorescence. b Boxplots showing mean and sample distribution for \% cells in the less oxidized population of replicates of (a). Sample size is $n=3$, being one each of independent cell lines 1-3 for WT, DM and TM, and triplicates of the MP cell line. The table shows ANOVA post-hoc Dunnet's T3 $p$ values for the indicated pairwise comparisons. c Boxplot of median fluorescent intensity (MFI) of the oxidized cell populations from (a). The horizontal dotted reference line and open arrow are identical to (A). The table shows ANOVA post-hoc Bonferroni $p$ values for the indicated pairwise comparisons

Contrary to the hypothesis that increased NNMT activity would reduce SAM levels as proposed for pluripotent human embryonic stem cells (hESCs) [52], SAM levels were not significantly altered by NNMT attenuation (Fig. S4D). We also treated DM cells with 1-MNA, which caused a morphological conversion from predominantly rounded to predominantly elongated form (Fig. S4E), suggesting that NNMT activity may contribute to the morphological differences between elongated MP and WT cells on the one hand and rounded DM and TM cells on the other. Other cell-types showed no significant change in cell morphology upon 1-MNA treatment (not shown).

\section{PGRMC1 phosphorylation status regulates genomic} methylation

Because of the reported effects of NNMT activity on global methylation levels [52], we assayed genomic CpG methylation levels. Hierarchical clustering of samples displayed striking separation of the cell groups, suggestive of highly divergent patterns of methylation among the cell types. All independently derived stable cell lines of each PGRMC1 state clustered closely to each other, and distant from other cell conditions (Fig. 7a).

We observed a highly significant number of differentially methylated probes (Fig. 7b). In the TM/DM comparison 


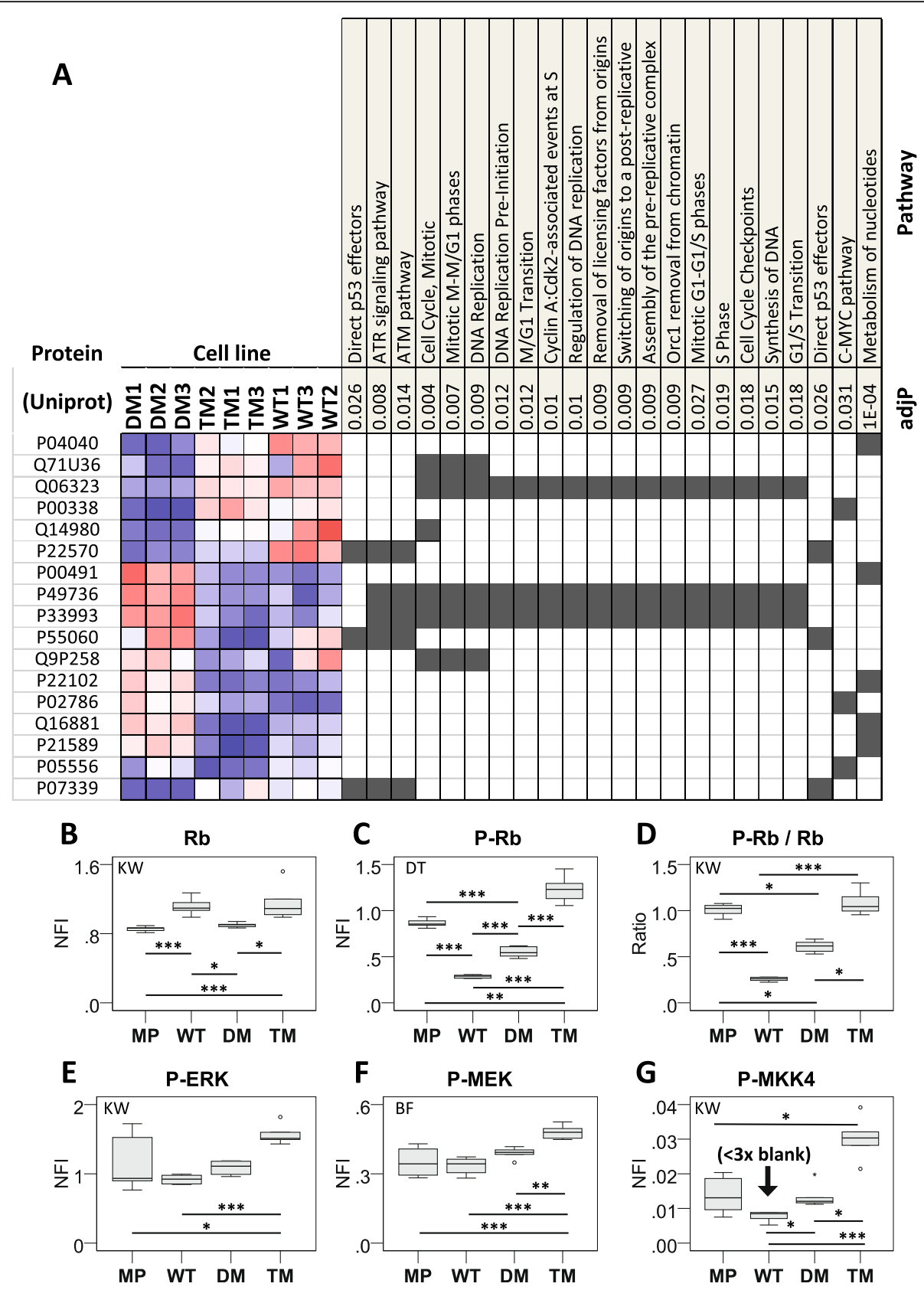

Fig. 4 Pathways enrichment suggests altered cell cycle control proteins. a Abundances of proteins detected as significantly differentially abundant in indicated Pathways Commons pathways by enrichment analysis (Accompanying paper [10]) at the adjP $<5 \%$ significance level. (b, $\mathbf{c}$, e-g) Average reverse phase protein array (RPPA) normalized fluorescent intensity (NFI) from the indicated antibodies (see methods) is plotted from 6 replicate measurements. NFI is normalized to protein content. Significance levels are ${ }^{*} p<0.05,{ }^{* *} p<0.01,{ }^{* * *} p<0.001$. Conventions follow those of the accompanying paper [10]. $\mathbf{d}$ The ratio of average NFI of the anti-pRB antibody to that of the RB antibody

(differ by Y180 state) we observed a shift towards hypermethylation that was not as pronounced in WT/MP (differ by transfection of pcDNA3-1_PGRMC1-HA and hygromycin stable selection) or DM/WT (differ by PGRMC1-HA S57/ S181 status). We then considered the percentage of differentially methylated probes which were hypermethylated in each comparison and noticed a clear increase in the progression from WT/MP, DM/WT to TM/DM (Fig. 7c).
While the WT/DM effect could be due to both PGRMC1HA expression and/or the stable antibiotic selection process, the differences between DM/WT and TM/DM were due solely to PGRMC1 mutation status. The overall level of CpG methylation was higher in TM cells across all chromosomes (Fig. 7c). Together, the results suggest a global increase in net methylation in this system, being highest in TM cells. 


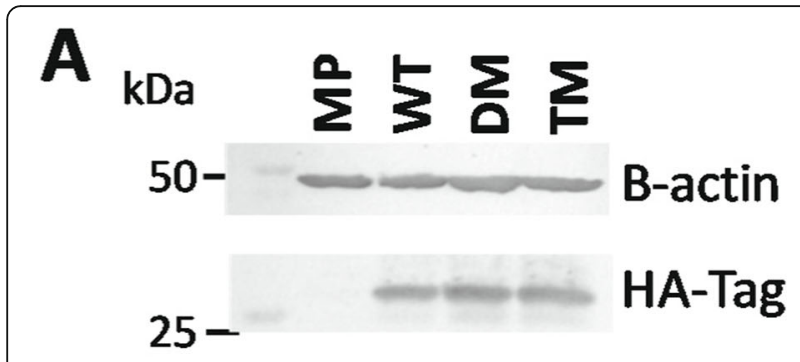

B Unique mutations/MB

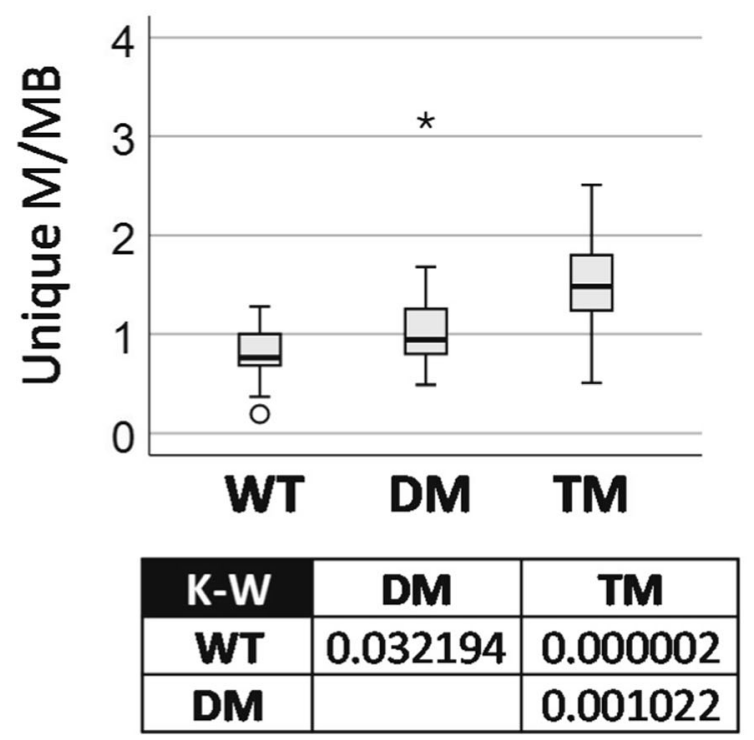

Fig. 5 PGRMC1 phosphorylation status affects DNA mutation rate. a Western blot of cells following thirty passages showing expression of HA-tag. Beta actin was used as a control. b Unique mutations per cell line relative to human reference genome and other genomes in the analysis. Mutations/MB are plotted for 23 chromosomes excluding $Y$. The box shows $p$ values for Kruskal-Wallis (K-W) posthoc pairwise comparisons

Significantly differentially methylated probes were next assessed by genomic context region based on the EPIC manifest designations for relation to $\mathrm{CpG}$ island (Island; Open Sea, North Shelf, North Shore, South Shore, and South Shelf) because DNA methylation levels are known to depend upon genomic context [54]. The percentage of significantly hypermethylated probes was similar for all PGRMC1-HA-mutation states (WT, DM, TM), which differed from MP (Fig. S7A, left). By contrast, there were marked differences in the distribution of hypomethylated probes due to PGRMC1-HA mutation status, with DM and especially TM cells exhibiting elevated levels of hypomethylation at $\mathrm{CpG}$ islands and both North and South Shore sites, and reduced levels of hypomethylation (increased methylation) in Open Sea regions (Fig. S7A, right). This is consistent with increased levels of Open Sea methylation (in excess to Island and Shore sites) leading to the net increased methylation of TM cells, with a minority of local demethylation sites in the vicinity of CpG Islands at activated genes. See also the volcano plot of Fig. 7b, TM/WT comparison, where positive fold change $(\log \mathrm{FC}>0)$ is in excess.

The increased level of hypomethylation at Islands and Shores in TM cells was not detectable between $5^{\prime}$ untranslated and 3' untranslated regions of annotated coding genes (Fig. S5). A proportion of cell-type and promoter-associated enhancers exhibited reduced hypermethylation or increased hypomethylation in the TM/ DM comparison (Fig. S6). This reduced level of enhancer methylation, normally associated with gene activation, occurred in the background of bulk overall increased CpG methylation in TM cells. Since TM and DM cells differ only in the presence of Y180 of the exogenous PGRMC1 protein, we conclude that Y180 phosphorylation status appears to specifically influence the genomic methylation status of cell-type specific enhancers.

We performed Kyoto Encyclopedia of Genes and Genomes (KEGG) pathways and Gene Ontology (GO) enrichment analysis of the methylomics results, using probes from Island or Shore genomics regions only. For KEGG we separated the data into separate hyper- and hypomethylated gene sets. The KEGG results are presented in Fig. S7B, and full GO and KEGG results are provided as File S1. Each mutation state induced specific unique suites of detected pathways. Most significantly differential KEGG pathways were detected by all three cell comparisons.

Across all comparisons, the most significant enriched GO terms included some associated with embryology and developmental processes (Table S2, File S1). KEGG results reflected the activities of signal pathways, cell-extracellular interactions, and cancer biology (Table S2, File S1). E.g., for the TM/DM cell comparison, which differs in only the presence of phosphate acceptor oxygen of PGRMC1-HA Y180, KEGG results indicated that the most hypomethylated pathway in TM cells was PI3K-Akt (path:hsa04151). The PI3/Akt pathway was also among the top 5 in the hypermethylated data set for that comparison, and was significantly differential in all three comparisons (File S1). This means that genes associated with PI3K/Akt activity exhibited altered methylation states. We observed no significantly different alteration of $\mathrm{CpG}$ methylation sites associated with the Akt gene itself (not shown). In the accompanying publication we predicted reduced PI3K/Akt activity in TM cells on the basis of proteomics pathways enrichment, where the pathway was also significantly differential for all cell comparisons, and we demonstrated reduced phosphorylation of Akt substrates in TM cells [10].

By way of illustrative example, we further explored the biology underyling the pathways analysis by considering the unique KEGG pathways identified for both hyper- 

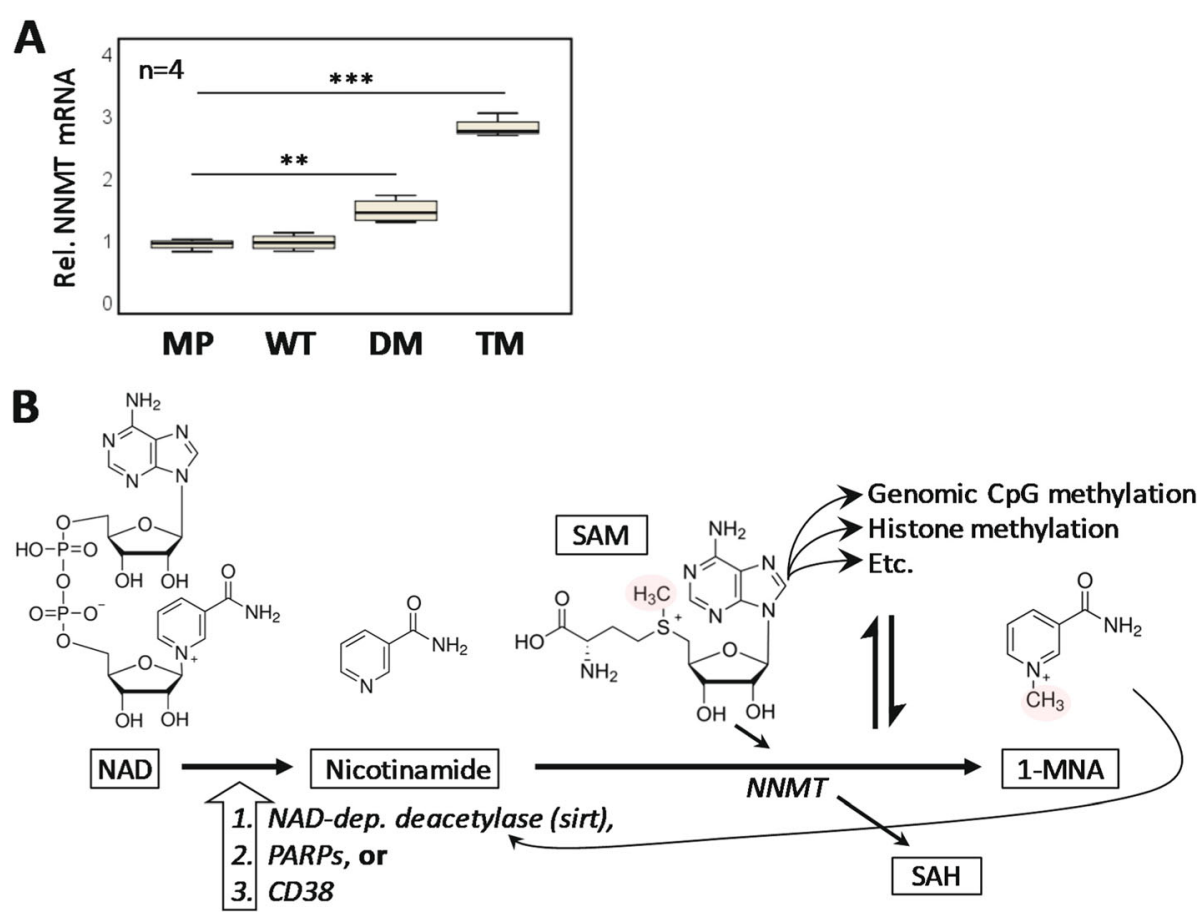

Fig. 6 PGRMC1 phosphorylation is associated with NNMT regulation. a Quantification of NNMT mRNA levels in the four cell types by RT-PCR. Quantification cycle $(\mathrm{Cq})$ results were quantified using the $2^{\Delta \Delta \mathrm{Cq}}$ method using beta actin as an internal control. ANOVA post-hoc Tukey's HSD $p<0.01\left(^{* *}\right)$ or $p<0.001\left(^{(* *)}\right.$. b Model of the NNMT and 1-methylnicotinamide pathway. Nicotinamide is produced from NAD by NADdependent deacetylases (e.g. Sirtuins), PARPS or CD38 [51]. NNMT transfers a methyl group of S-adenosylmethionine (SAM) to nicotinamide to produce 1-methylnicotinamide (1-MNA), producing S-adenosyl homocysteine (SAH). This reaction is in competition with methylation reactions including DNA and histone methylation by SAM [52]. 1-MNA can feed back to stabilize sirtuin 1 [53]

and hypo-methylated genes from Fig. S7A. 13 hypermethylated and 7 hypo-methylated pathways were unique to the TM/DM comparison (Fig. S7B). Their identities are given in Table S3. Hyper-methylated pathways included processes related to proteolysis and bacterial infection as well as DNA base excision repair and mismatch repair involved in averting mutations. The latter is striking because we also detected protein abundances involved in pathways associated with DNA damage (p53 effectors, and ATM/ATR) to be reduced in TM cells by proteomics (Fig. 4), with accompanying elevated mutation rate in TM cells (Fig. 5), which is fully consistent with the methylomics pathways biology. These circumstances suggest strongly that the study reflects true biology related to PGRMC1 Y180 function.

Whereas NNMT was more abundant in TM cells (Fig. 6a), CpG sites in its gene were hypermethylated (not shown) as were the sites in many coding genes (Fig. S5). Histone methylation status, which we have not assayed, could be influential in determining gene expression levels, or a hypomethylated enhancer may activate transcription despite CpG methylation of the immediate gene locus. Approximately $80 \%$ of the genes encoding the 243 differential proteomics heat map of the accompanying paper [10] were significantly differentially methylated between one of the comparisons (not shown), suggesting that epigenetics may contribute greatly to the phenotypes observed for these cell types.

\section{Discussion}

Our data shows that changes in posttranslational modification of PGRMC1 result in altered metabolism, genomic mutation rates, and epigenetic methylation status. Future research should examine whether genomic effects involve previously reported PGRMC1 involvement with G0/G1 checkpoint and/or spindle association [2, 20-24].

Our most important finding is that all tested PGRMC1 conditions induced specific fingerprint patterns of genomic CpG methylation, identifying PGRMC1 as a major epigenetic regulator. In particular, PGRMC1 Y180 affected pathways predicted to be associated with embryology, seemingly by targeting the genomic methylation status of $\mathrm{CpG}$ sites in a subset of cell-type and promoter-associated enhancers. Vertebrate embryology relies heavily on epigenetic histone and genomic methylation to direct cell plasticity between generations and in response to stimuli to determine the differentiation status of cells [55]. Y180 (and Y139) appeared in animal evolution in the common ancestor of eumetazoans (cnidarians, including corals, jellyfish, etc., and bilaterally symmetrical animals), concomitantly with the gastrulation 

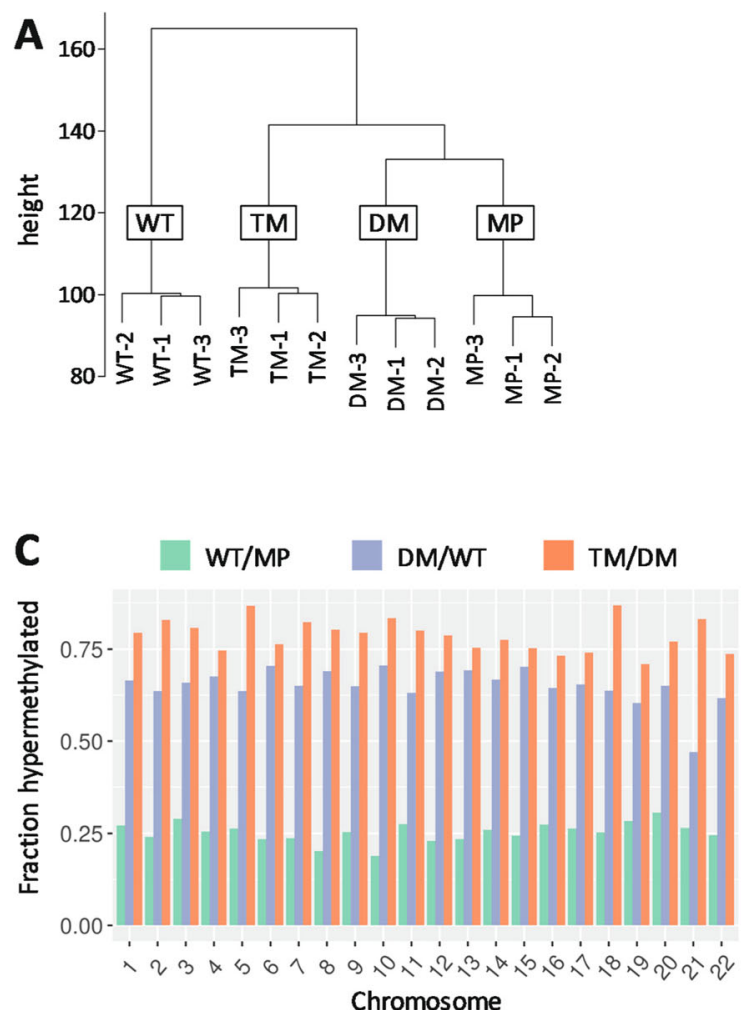

Fig. 7 PGRMC1 phosphorylation mutants elicit distinct patterns of genomic methylation. a Hierarchical clustering of methylation data from all cell lines sampled in triplicate. Clustering was applied using 520,031 probes with a coefficient of variation $>0.10$. Height is calculated using the UPGMA method in the Lumi package and equates to the average distance between two members of two groups. $\mathbf{b}$ Volcano plots of the WT/MP, DM/WT and TM/DM comparisons. The log2 fold change (FC) ( $x$ axis) is plotted relative to the log $10 p$-value ( $y$ axis) to visualize the magnitude of the most significant features. Probes with an adjusted $p$-value $<0.05$ and absolute log FC $>1$ are plotted in green. Probes with an absolute logFC $>1$ that were not significant are plotted in orange. Probes with a logFC $<1$ that were significant at adjusted $p$-value $<0.05$ are plotted in blue. Black probes were not significant. c Significant probes in each differential methylation comparison were extracted and the fraction of hypermethylated or hypomethylated probes per chromosome calculated. Methylation levels are higher in TM cells across all chromosomes

organizer that drives the embryological tissue differentiation processes [12]. PGRMC1 Y180 was therefore evolutionarily acquired before the appearance of chordate organs, and the embryological differentiation processes that give rise to them, which immediately suggests how PGRMC1 may affect cancer cell plasticity. Differential PGRMC1 phosphorylation status may therefore be associated with the altered prognosis of triple negative breast cancers [9].

The increase of hypermethylated CpG sites observed in TM cells (Fig. 7, Fig. S5) is indeed reminiscent of the characteristic increase in hypermethylation associated with both hESCs and induced pluripotent stem cells (iPSCs). The genome becomes increasingly hypomethylated during embryogenesis and somatic tissue differentiation [56]. It is as if the maintenance of vertebrate differentiation processes depended upon the acquisition of Y180 and the organizer by the eumetazoan common ancestor [12], and, upon disturbance with Y180 functions in TM cells, the genomic CpG methylation pattern reverts to a phenotype reminiscent of the undifferentiated state of that ancestral evolutionary stage: prior to the appearance of deuterostome and chordate tissues and body plan. This phenomenon clearly merits future investigation.

NNMT regulates pluripotency in naïve hESCs, where it was proposed to deplete cellular SAM levels and pleiotropically lead to reduced histone methylation, including some genes in the Wnt pathway whose concomitant elevated expression affected pluripotency [52]. PGRMC1 involvement in hESC pluripotency [57] suggests that it could be mechanistically involved in the epigenetic modulation that regulates hESC pluripotency [52]. Perhaps the most striking result we observed was that each PGRMC1 phosphorylation mutant condition elicited a distinctly unique pattern of genomic methylation (Fig. 7), consistent with PGRMC1 playing a central role(s) in regulating genomic methylation, with each phosphorylated isoform capable of dynamically engendering different end-points. 
As a target of Wnt signaling, TCF/LEF appeared very early in animal evolution, being absent from the single-celled choanoflagellate sister group to metazoans, but present in sponges [58]. By the time the common ancestor of eumetazoans evolved, embryological gastrulation initiated from the animal pole under control of Wnt//-catenin signaling, inducing an axial organizer whose vertebrate descendant is known as the Spemann-Mangold organizer in amphibians. This initiates the orchestrated series of events where specific gene programs are activated by transcription factors that trigger differentiation into specialized cell types [58-61].

These processes must represent evolutionary novel metazoan functions. The correlation of appearance of the Y139/Y180 combination and organizer function [12] is consistent with the involvement of PGRMC1 in this process. This new hypothesized PGRMC1 functionality would seem to be superimposed upon more ancient PGRMC1 functions, such as sterol synthesis and steroid responsiveness. In support of this hypothesis, despite dramatic differences in energy metabolism, cell morphology, and epigenetics, all of our mutants responded similarly to P4-dependent resistance to doxorubicininduced death, which requires PGRMC1::cyP450 interactions [11], as well as to AG-205 toxicity (Fig. 2).

PGRMC1 is required to maintain the pluripotency of embryonic stem cells by influencing the p53 and Wnt/ $\beta$-catenin pathways [57]. In the accompanying publication [10] we show that TM cells exhibit lower levels of PI3K/Akt activity, and lowered levels of GSK3- $\beta$ phosphorylation on an Akt target site. The canonical Wnt pathway is activated when $\beta$-catenin translocates to the nucleus to act as a transcription factor after activation by Wnt ligands. Active GSK3- $\beta$ phosphorylates $\beta$-catenin, targeting it for polyubiquitination and proteasomal degradation [62], and Akt phosphorylation of GSK3- $\beta$ inhibits GSK3- $\beta$ activity [62].

While our cells are not embryonic, lack of Y180 phosphorylation and low PI3K/Akt activity could correlate with increased GSK3- $\beta$ activity, and lower $\beta$-catenin levels which would attenuate Wnt signaling via TCF/LEF. PGRMC1 has been associated with negative regulation of TCF/LEF in hGL5 granulosa/luteal cells [63] and hESCs [57]. Future experiments are required to explore this concept further in this and other cell models, which should be prioritized.

The propensity for animals and their cells to age was accompanied in evolution by the loss of pluripotent stem cells in the adult: an evolutionary necessity to establish larger collections of clonally communal but functionally specialized cells with diverse differentiation states [64]. That entire biology was associated with the evolution of the tissue and cell-type heterogeneity of bilateral animals, which started with the evolutionary appearance of the organizer [64]. The possible involvement of PGRMC1/PGRMC2 phosphorylation in cell cycle control mechanisms governing entry to $\mathrm{G}_{0}$ and senescence $[20,21,24,65]$ may accordingly be related to an overarching function associated with the reduction of the immortality potential of primitive eukaryotes, and the necessary evolution of a systematics of differentiation status and replicative control in bilateral animals [64]. This hypothesis merits future examination.

The NNMT pathway has been linked to lifespan and aging [66-69], to sirtuin protein stabilization in positive a feedback loop to the pathway in Fig. 6b involved in energy regulation and obesity $[52,70,71]$ and to epigenetics in cancer and stem cell status [52, 72-74] (reviewed by [75]). We found that TM showed increased 1-MNA indicating increased NNMT activity, and implicating PGRMC1 Y180 in modulation of this system.

In mining our proteomics data from the accompanying manuscript [10] for enzymes related to the NAD/ NNMT/1-MNA pathway (Fig. 6b), we noted that nicotinamide phosphoribosyltransferase (NAMPT) was one of the relatively few proteins less abundant in TM compared to both DM and WT (Fig. S3). NAMPT catalyzes a competing reaction to NNMT, whereby nicotinamide is converted to nicotinamide mononucleotide, which is then transformed into $\mathrm{NAD}^{+}$by nicotinamide/nicotinic acid mononucleotide adenyltransferase. NAMPT is the rate-limiting enzyme in this pathway, and regulates sirtuin activity by modulating $\mathrm{NAD}^{+}$levels [76]. This entire pathway is related to aging processes [77], which were acquired by bilaterian animals along with tissue differentiation and cell cycle control mechanisms to evade cancer [64]. Indeed, these are the very types of biology with which PGRMC1 function is here implicated: suggesting that PGRMC1 phosphorylation could be involved in the mechanism(s) of animal aging. Considering the long coevolutionary history of bilaterians and PGRMC1 tyrosine phosphorylation sites, this merits future study.

Our results imply that the ability to interchange between phosphorylated states at T178, Y180, S181 and S190 [5] will be critical in supplying correct PGRMC1 function at appropriate times for the cell cycle and state of cell differentiation during early embryology. Clearly, Y180 is important for PI3K/Akt activity [10], a major pathway associated with aging [78].

As a final technical note, S181 has been discussed in the literature as a consensus CK2 site [2]. It must be noted in this context that PGRMC1 phosphorylation at S181 was unaffected by knockout of CK2 kinase activity in C2C12 mouse myoblast cells [79], indicating that at least in those cells CK2 was not responsible for S181 phosphorylation. Willibald et al. [80] recently used an antibody against PGRMC1 phospho-S181 (pS181) to argue that S181 phosphorylation is essential in PGRMC1 activation, as well as discussing the presence of phosphorylated PGRMC1 in immunohistochemistry of tissue biopsies [81]. A caveat to these results is that the peptide epitope used to generate the antibody (which was designed and commissioned by 
M.A.C. while at ProteoSys AG, $[9,82])$, corresponded to a CK2 consensus site. CK2 is a promiscuous kinase which gives rise to $10-20 \%$ of the human phosphoproteome [83]. On Western blots of breast cancer whole cell proteins, that antibody generated a low background smear of signals across many molecular weights, in addition to recognizing exogenously expressed PGRMC1 but not a S181A mutant (not shown). More rigorous characterization of the antipS181 antibody specificity would be required to exclude that the immunohistochemistry of tissue biopsies was not detecting increased levels of non-PGRMC1 CK2 phosphorylation sites.

\section{Conclusion}

Our work was inspired by the discovery that PGRMC1 phosphorylation differs between breast cancer subtypes differing in estrogen receptor expression, that also exhibit starkly contrasting levels of patient survival [9]. It is wholly feasible that characterization of the signal network surrounding PGRMC1 could lead to novel treatments for cancer, and other diseases involving PGRMC1 [2]. This work suggests that PGRMC1 is a key yet hitherto unrecognized protein involved in the early evolution of animals, and that its phosphorylation status is capable of influencing biological processes which can destabilize the ordered harmony of eumetazoan multicellular organismal collections of functionally specialized cells, with imperatively relevant connotations for human disease.

\section{Methods}

\section{Establishment of cell lines and cell culture}

Construction of cell lines and culture conditions is described in the companion publication [10]. Briefly, pcDNA3.1 plasmids expressing either a wild-type PGRMC1 open reading frame with a $3 \mathrm{x}$ heamaglutinin (3HA) tag (WT), an S57A/S181A double mutant (DM) or an S57A/ Y180F/S181A triple mutant (TM) were transfected into MIA PaCa-2 cells and stably selected by hygromycin selection. Three independent cell lines were established for each plasmid, and equivalent PGRMC1-HA expression levels for each line were established by Western blot. The exogenous PGRMC1-HA protein is present at approximately the same levels as endogenous MP cell PGRMC1. Individual cell lines have not been clonally selected, and so probably represent mixed lineages.

\section{NpFR1 measurement of cytoplasmic redox status}

Intracellular redox state measurement by fluorescent redox sensor NpFR1 was performed by flow cytometry as described for NpFR2 [10].

\section{Genomic DNA isolation and sequencing}

Genomic DNA for sequencing was isolated from one subline of each cell condition. Passaging was repeated every 4 days for thirty passages, with media changes every $48 \mathrm{~h}$. To extract genomic DNA after thirty passages, approximately $5 \times 10^{6}$ cells were centrifuged for $5 \mathrm{~min}$ at $300 \times \mathrm{g}$. The cells were suspended in $200 \mu \mathrm{L}$ phosphate buffer saline. The purification of total DNA from cells was performed using the DNeasy Blood \& Tissue Kit (Qiagen, C.N 69504) following the manufacturer's protocol. The concentration of the dsDNA was measured using a Qubit dsDNA Hs Assay Kit (Life Technologies, Ref Q32851). The samples were sent to Kinghorn Centre for Clinical Genomics (Garvan Institute of Medical Research, 384 Victoria St, Darlinghurst, NSW 2010 Australia) for whole genomic sequencing. DNA for methylation analyses was extracted the same way except we used passage 10 cell lines, consisting of triplicate cultures of MP cells, and respective lines 1-3 of each PGRMC1-HA mutant condition (WT1, WT2, WT3, DM1, DM2, DM3, TM1, TM2, TM3).

\section{MTT cell viability assays for effects of P4 on the dox and AG-205 responses}

To assay for proliferative and protective effects of $\mathrm{P} 4,10^{4}$ cells were seeded per 96 well plate well. For each cell type, $n=6$ replicates were allowed to adhere for $3 \mathrm{~h}$ after which viable adherent cells were quantified by 3-(4,5-dimethylthiazolyl-2)-2,5-diphenyltetrazolium bromide (MTT) assay. Previous experiments had shown adherence to be complete after $1.5 \mathrm{~h}$ (data not shown). Identical replicates were incubated overnight in complete DMEM. After $23 \mathrm{~h}$ the media was exchanged for complete DMEM containing either $1 \mu \mathrm{M}$ P4 or DMSO vehicle control. After a further $1 \mathrm{~h}$ incubation (t0) the media was exchanged for media containing either $1 \mu \mathrm{M}$ P4 or DMSO vehicle control (to assay for proliferative effects, Fig. 2a), or with varying concentrations of doxorubicin (dox) (Fig. 2b-c) or AG-205, +/- P4 [36]. Cells were incubated for a further $24 \mathrm{~h}$ followed by viable cell quantification by MTT assay. The media was discarded, cells were washed with warm PBS and incubated with $100 \mu \mathrm{L}$ of $0.5 \mathrm{mg} / \mathrm{mL}$ MTT (Sigma-Aldrich M2128) in phenol red free media (Sigma-Aldrich D1145). After $3 \mathrm{~h}$ incubation at $37^{\circ} \mathrm{C}$ and $5 \% \mathrm{CO}_{2}$, the media was removed and $100 \mu \mathrm{L}$ of DMSO (EMD Millipore, 317,275) was added to each well to solubilize the Formazan crystals. The cells were incubated for $1 \mathrm{~h}$ followed by mixing, and absorbance was read at $570 \mathrm{~nm}$ using a plate reader (Bio-Strategy $\mathrm{P} / \mathrm{L}$, Campbellfield, Vic., Australia). The percentage of viable cells was estimated by normalizing the absorbance of the treated or untreated cells to the average values from panel A.

\section{NNMT shRNA attenuation}

We established an anti-NNMT sh-RNA-expressing TM cell line by lentiviral transduction and puromcyin stable selection. Anti NNMT shRNA lentiviral production was as described using mission TRC2-pLKO-Puro series Lentiplasmid (SHCLND, Sigma-Aldrich) scramble shRNA (scr- 
sh) [10] and shRNA targeting NNMT (Sigma-Aldrich TRCN0000294436, TGCAGAAAGCCAGATTCTTAA). WT and TM cell lines expressing either scrambled shRNA (scr-WT, scr-TM) or anti NNMT shRNA (shNNMT-WT, shNNMT-TM) were seeded in 24 well plates until they reached approximately $50 \%$ confluency followed by transduction with shRNA virus particles and selection as described [10].

\section{RT-PCR}

RNA was extracted by total RNA mini kit (Bio-Rad, 7, 326,820 ) according to the manufacturer's instructions. cDNA was synthesized in C1000 Touch $^{\text {Tx }}$ Thermal Cycler using cDNA synthesis kit (Bio-Rad, 1,708,890) following Bio-Rad's recommended protocol. Real-time qPCR was performed on a CFX96 Touch $^{\text {ma }}$ Real-Time PCR Detection System utilizing iTaq ${ }^{\mathrm{mm}}$ Universal SYBR ${ }^{\circ}$ Green Supermix (Bio-Rad, 1,725,120). NNMT Primers for qPCR were synthesized at Monash University and the sequences were obtained from [84]. NNMT primers used in this experiment were Forward sequence 5'GAATCAGGCTTCACCTCCAA-3' and Reverse sequence 5'-CCCAGGAGATTATGAAACACC-3'. Actin was used as an internal control Forward sequence 5'GACGACATGGAGAAAATCTG-3' and Reverse sequence 5'ATGATCTGGGTCATCTTCTC-3'.

\section{Western blot}

Cells were lysed with radioimmunoprecipitation assay buffer (RIPA buffer) (Sigma Aldrich, R0278) supplemented with protease and phosphatase inhibitor cocktail (Thermoscientific, 88,668). After scraping the cells, they were centrifuged at $8000 \mathrm{rpm}$ for $20 \mathrm{~min}$ (Hermle Centrifuge $\mathrm{Z} 233 \mathrm{M}-2)$ at $4{ }^{\circ} \mathrm{C} .20 \mu \mathrm{g}$ protein of thirty passage number of MP, WT, DM, and TM were loaded into the wells of a $12.5 \%$ SDS-PAGE gel and set to run at $150 \mathrm{~V}$ for $45 \mathrm{~min}$ on Power Pac. Transferring the protein from the gel to a PVDF membrane occurred under wet transfer with $1 \mathrm{x}$ Towbin buffer at $40 \mathrm{~V}$ for $3 \mathrm{~h}$ in an ice bath. The membranes were incubated with 1:2000 diluted Beta-Actin (Sigma Aldrich, AS441) and 1:2000 HA-Tag (Sigma Aldrich, H3663) primary antibodies in blocking buffer overnight at $4{ }^{\circ} \mathrm{C}$ with shaking. Next day, the membranes were washed twice with $0.05 \%$ PBS-T and incubated with antimouse secondary antibody; horse radish peroxidase (HRP), for $1 \mathrm{~h}$ at room temperature. Next, the membranes were washed twice with $0.05 \%$ PBS-T and once with PBS. Colorimetric detection of the bands occurred using tetramethylbenzidine as described [10].

\section{Reverse phase protein array}

Methods for reverse phase protein arrays are described in the accompanying publication [10]. The following primary antibodies (provider and reagent number, dilution) were used here: Erk1/2-P-Thr202/Tyr204 (CST 4370, 1: 100), MEK1/2-P-Ser217/Ser221 (CST 9154, 1:100), MKK4(SEK1)-P-Ser257/Thr261 (CST 9156, 1:100), Rb (CST 9309, 1:200), Rb-P-Ser807/Ser811 (CST 8516, 1: 100).

\section{Hyperspectral fluorescence microscopy}

UV and visible continuous wave epifluorescence microscopy with multiple excitation wavelength ranges from $335 \mathrm{~nm}$ to $532 \mathrm{~nm}$ and measuring emission in three emission wavelength ranges $450+/-30 \mathrm{~nm}, 587+/-17.5$ $\mathrm{nm}$ and $700 \mathrm{~nm}$ long pass were used. Excitation wavelengths are supplied by low cost multi-LED light source optical fibre coupled to the Olympus IX71 microscope. An Andor/Oxford Instruments iXon 885 Electron Multiplying Charged Coupled Device (EMCCD) was used to capture images typically using an EM gain sufficient to lift the low light auto-fluorescence signal above the 17 electrons per pixel per second readout noise but with minimal contribution from clock induced charge. Gain linearity is ensured by using the Real Gain ${ }^{\text {tw }}$ technology (Andor/Oxford Instruments). The camera is operated below $-70^{\circ} \mathrm{C}$ such that thermal noise is negligible. Quantum efficiency of the sensor varies from 50 to $65 \%$ across the range of interest from 450 to $670 \mathrm{~nm}$ and the intensity is digitized into $\sim 16 \mathrm{~K}$ values. Background signal is subtracted from all images which is kept minimal through the use of low fluorescence petri dishes (CELLview, Greiner Bio-One). A single wavelength image may take between 1 and $5 \mathrm{~s}$ depending on the sample and wavelength but $1-2 \mathrm{~min}$ is typically required for the entire stack of images for all spectral channels,, where the features for Fig. 1a were as follows.

Feature 1: 'Ratio of Channel 4 to Channel 10', Feature 2: 'Ratio of Channel 9 to Channel 16', Feature 3: 'Ratio Channel 2 to Channel 16', Feature 4: 'Ratio of Channel 5 to Channel 14', Feature 5: 'Ratio of Channel 2 to Channel 7', Feature 6: 'Ratio of Channel 9 to Channel 18', Feature 7: 'Ratio of Channel 7 to Channel 15', Feature 8: 'Ratio of Channel 7 to Channel 9',

A description of the channels used and their spectral ranges is given in Table S1. During growth under standard mid-log phase growth conditions, hyperspectral imaging was performed on $\sim 300$ live cells per cell condition (MP, WT, DM, TM) and the mean cellular intensity in each channel was calculated. Pixel correlations between spectral channels were removed using principal component analysis (PCA), followed by a discriminatory projection to maximally separate the four cell groups (Fig. 1a). Technical details of this approach are described by Gosnell et al. [44]. In order to statistically analyze the cluster separations of Fig. 1a, an additional LDA projection of cell data was carried out, this time with two classes of cells chosen 
at a time. Their average spectra were projected onto a common direction identified by this additional LDA. The cell distributions were then tested by the KolmogorovSmirnov test.

\section{Metabolite preparation}

Scr-WT, scr-TM, shNNMT-WT, and shNNMT-TM cells as generated in "5.5. NNMT shRNA attenuation" above were seeded in Greiner Bio-One Tissue Culture Petri Dish $100 \mathrm{~mm} \times 20 \mathrm{~mm}$ (Interpath, 664,160). The cells were rinsed with pre-warmed deionised water with brief shaking. The plates were placed on liquid nitrogen for $15 \mathrm{~s}$. Cells were extracted with $1 \mathrm{~mL}$ of ice-cold extraction solvent (2:2:1methanol-ethanol-water) and harvested with scraper. The contents were transferred to $1.5 \mathrm{~mL}$ Eppendorf tube. The tubes were centrifuged at $4{ }^{\circ} \mathrm{C}$ for $5 \mathrm{~min}$ at $16,100 \mathrm{x} \mathrm{g}$ and supernatant were transferred to a new $1.5 \mathrm{~mL}$ Eppendorf tube. The supernatant was filtered and evaporated dry under a gentle stream of nitrogen gas. The residual precipitates were resuspended in $500 \mu \mathrm{L}$ of $60 \%$ acetonitrile/water $\left(\mathrm{ACN} / \mathrm{H}_{2} \mathrm{O}\right)$ containing $\mathrm{m}$-tyrosine $(10 \mu \mathrm{g} / \mathrm{mL})$ (Sigma, Castle Hill, NSW) as an internal standard.

\section{Metabolite quantification}

Metabolomic analysis was performed using an Agilent 1290 Infinity HPLC system equipped with a quaternary pump, degasser, temperature-controlled column and sample compartment coupled to an Agilent 6470 triple quadrupole (QQQ) mass spectrometer with a Jet Stream electrospray ionization (ESI) source (Agilent Technologies, Australia).

The column temperature was maintained at $35^{\circ} \mathrm{C}$ and the autosampler temperature was set at $4{ }^{\circ} \mathrm{C}$. The electrospray (ESI) source settings were as follows: nebulizer gas, 45 psig; drying gas flow rate, $8.0 \mathrm{~L} / \mathrm{min}$; drying gas temperature, $300^{\circ} \mathrm{C}$; sheath gas temperature, $350^{\circ} \mathrm{C}$, sheath gas flow, 10 $\mathrm{L} / \mathrm{min}$; capillary voltage, $4000 \mathrm{~V}$; and nozzle voltage, $0 \mathrm{~V}$. The data were acquired positive ionization mode.

Methylnicotinamide samples and samples from Fig. 6a were analyzed with a Kinetex HILIC column $(50 \mathrm{~mm} \times$ $2.1 \mathrm{~mm}, 2.6 \mu \mathrm{m}$ particle size, pore size of $100 \AA$, Phenomenex, CA, USA). The mobile phases were $100 \%$ HPLC-grade acetonitrile (A) and $10 \mathrm{mM}$ ammonium acetate in HPLC-grade water adjusted to $\mathrm{pH} 3$ with formic acid (B) at a flow rate of $200 \mu \mathrm{L} / \mathrm{min}$. The solvent gradient began with $95 \% \mathrm{ACN}$ for $0.5 \mathrm{~min}$, decreasing linearly to $35 \% \mathrm{ACN}$ over $12 \mathrm{~min}$. The gradient was maintained at $35 \% \mathrm{ACN}$ for $0.5 \mathrm{~min}$ and increased to 95\% ACN over $0.1 \mathrm{~min}$. The mobile phase composition was then held at $95 \% \mathrm{ACN}$ for $10 \mathrm{~min}$ to re-equilibrate the column before the next injection. The total time for the gradient program was $24 \mathrm{~min}$.
1-MNA was tentatively identified with $\mathrm{m} / \mathrm{z}$ of 137.0633 $\mathrm{Da}$, and an MS/MS score of 99.74. Samples of authentic 1methylnicotinamide chloride (1-MNA) (CAS Number 1005-24-9, Sigma-Aldrich \# M4627), 2-methylnicotinamide (2-MNA) (CAS Number 58539-65-4, Ark Pharm Arlington Heights, IL 60004, USA, \#AK-39636) and Nmethylnicotinamide (N-MNA) (CAS Number 114-33-0, Sigma-Aldrich \#M4502) were injected at three concentrations $(1,10$ and $100 \mu \mathrm{g} / \mathrm{mL})$ to confirm identification of 1MNA. The compound was unambiguously identified as 1MNA with retention time (RT) of $7.33 \mathrm{~min}$ and $\mathrm{m} / \mathrm{z}$ of 137.0717, and not 2-MNA (RT $0.96 \mathrm{~min}, \mathrm{~m} / z$ 137.0719) or N-MNA (RT $0.97 \mathrm{~min}, \mathrm{~m} / z$ 137.0709). The response of the triple quadrupole mass spectrometer to 1-MNA was linear over the range of $10 \mathrm{ng} / \mathrm{mL}$ to $1 \mu \mathrm{g} / \mathrm{mL}$, and the identity was confirmed by MS/MS and HPLC retention time using 2-methylnicotinamide and $\mathrm{N}$-methylnicotinamide standards (not shown).

Cellular extracts except Fig. 6a were analyzed with a HILIC-Z column $(50 \mathrm{~mm} \times 2.1 \mathrm{~mm}, 2.6 \mu \mathrm{m}$ particle size, $100 \AA ̊$ pore size, Agilent Technologies, CA, USA) equipped with a guard column of the same stationary phase. The mobile phases were HPLC-grade acetonitrile:water (95:5) (A) and water (B), both containing $20 \mathrm{mM}$ ammonium formate and $0.1 \%$ formic acid $(\mathrm{pH} \sim 3$ ) at a flow rate of $400 \mu \mathrm{L} / \mathrm{min}$. The solvent gradient started at $100 \% \mathrm{~A}$ and was held for $0.5 \mathrm{~min}$, followed by a linear gradient from $100 \%$ A to $60 \%$ A over $9 \mathrm{~min}$. The gradient was maintained at $60 \% \mathrm{~A}$ for $0.5 \mathrm{~min}$ and returned to $100 \%$ A over $0.1 \mathrm{~min}$. The solvent was then held at $100 \% \mathrm{~A}$ for $5.4 \mathrm{~min}$ to re-equilibrate the column before the next injection. Injection volume was $2 \mu \mathrm{L}$. The total time for the gradient program was $15 \mathrm{~min}$.

Analysis of the set of samples was preceded and followed by injection of five concentrations of 1-MNA $(10 \mu \mathrm{g} / \mathrm{mL}$ to $1 \mathrm{ng} / \mathrm{mL}$ in log steps) and six concentrations of S-adenosyl methionine (SAM) $(100 \mu \mathrm{g} / \mathrm{mL}$ to 1 $\mathrm{ng} / \mathrm{mL}$ ) (Sigma-Aldrich, Castle Hill, NSW). In addition, after every 15 samples a blank injection and a mixture of 1-MNA, m-tyrosine and SAM was run to verify that there was no carryover between samples and that retention time and detector sensitivity had not changed during the course of running the samples.

The selective detection and quantitation of 1-MNA was achieved by monitoring the transition of $\mathrm{m} / \mathrm{z} 137 \rightarrow 108$ using a fragmentor voltage of $120 \mathrm{~V}$ and collision energy of $20 \mathrm{~V}$, with the further requirement that retention time matched that of the authentic compound (ca. $2.7 \mathrm{~min}$ ). mtyrosine was quantitated by monitoring the transition $\mathrm{m} / \mathrm{z}$ $182->136$ (fragmentor voltage $135 \mathrm{~V}$, collision energy 20 $\mathrm{V}, \mathrm{RT}=4.9 \mathrm{~min}$ ) and SAM was quantitated by monitoring the transition $m / z 399$ - $>136$ (fragmentor voltage $100 \mathrm{~V}$, collision energy $20 \mathrm{~V}, \mathrm{RT}=9.1 \mathrm{~min}$ ). The fragmentor voltage and collision energy values were determined by optimizing system response to authentic 1-MNA, m-tyrosine 
and SAM. Limits of quantitation were determined by regressing the $\log$ (detector response) to $\log$ (concentration) response over the range of serial dilutions for each standard, and by observing where the dose/response curve departed from linearity.

\section{Effect of 1-MNA on morphology}

MiaPaCa-2 cells expressing DM PGRMC1-HA were seeded at $25 \%$ confluency and cultured overnight. The media was removed and the cells were incubated in the presence of the indicated amount of 1-MNA (Sigma-Aldrich, Castle Hill, NSW) for $24 \mathrm{~h}$. The 1-MNA stock was made in sterile $\mathrm{H}_{2} \mathrm{O}$. Five random images were taken per treated culture and assigned random numbers 1-20. These were given to a blinded scorer who decided for each image whether given cells were round or elongated.

\section{Methylome assay}

Genomic DNA from each cell subline were processed using the Illumina Infinium HD Methylation Assay (EPIC array) which interrogates $>850,000 \mathrm{CpG}$ sites. The four cell lines were processed in triplicate equating to 12 samples, being three replicates of MP cells, and independent cell lines 1-3 for each of the PGRMC1 states [10]. The array was prepared at the Australian Genome Research Facility (AGRF) following the manufacturer's instructions. Quality checking of the samples was performed by Nanodrop Spectrophotometer and resolution on a $0.8 \%$ agarose gel at $130 \mathrm{~V}$ for $60 \mathrm{~min} .500 \mathrm{ng}$ total DNA was bisulfite converted with Zymo EZ DNA Methylation kit (Zymo Research, Irvine, CA, USA), following the manufacturer's standard protocol. Amplified DNA samples were fragmented and resuspended DNA was loaded onto a BeadChip. The BeadChip was incubated overnight while DNA fragments anneal. After hybridization the array was imaged on the Illumina iScan system.

Methylation data were processed using in $\mathrm{R}$ version 3.5.1 (www.r-project.org). Data were assessed using the hg19 build of the human referene genome annotated in the IlluminaHumanMethylationEPICanno.ilm10b4.hg19 bioconductor annotation. Quality control of the array was assessed with the R package Lumi [85]. Probes on the sex chromosomes, cross-reactive probes and probes with known SNPs at the CpG site were excluded. Following filtering 812,817 probes remained. The cleaned data were normalized with the SWAN normalization, and sample relationships examined. Genome-wide differential methylation analysis was undertaken using Limma [86]. Multiple testing correction was applied using the Benjamini and Hochberg method. Differential methylation in each group was observed on volcano plots and heatmaps.

Due to the excessive quantity of differentially methylated probes with small effects we refined the data used for functional analysis to the top 2000 most variable probes prior to pathways analysis. These probes explained $>90 \%$ of the total variance among the first two principle components while preserving a reasonable number of intragenic probes. Pathways enrichment was undertaken in $\mathrm{R}$ using the enrichPathway function in the ReactomePA package [87], which implements a onetailed hypergeometric test for overrepresentation. Multiple testing adjustment was applied with the Bonferroni \& Hochberg FDR. KEGG pathways analysis was undertaken using the kegga function in the $\mathrm{R}$ package in LIMMA. Enrichment was calculated for genes from the top 2000 most variable probes with a $p$-value $<0.05$. The gene sets were split to those with hypomethylated probes $(\log \mathrm{FC}<0)$ and those with hypermethylated probes $(\log \mathrm{FC}>0)$ for enrichment. Enrichment of Gene Ontology (GO) terms was undertaken using the GOstats package [88]. Ontology assignments are calculated using a hypergeometric test for overrepresentation on the significantly differentially methylated probes $(p<0.05)$ among the 2000 most variable. Multiple testing adjustments were applied using the FDR. Intersects of common pathways from KEGG and Reactome analyses were assessed using the UpSetR package [89].

\section{Supplementary information}

Supplementary information accompanies this paper at https://doi.org/10. 1186/s12860-020-00268-z.

Additional file 1 Fig. S1. Representative hyperspectral autofluorescence cell images from the measurements. Related to Fig. 1. (A) Mean cellular intensity of hyperspectral autofluorescence channel 18 [495 nm(Ex), 700 $\mathrm{nm}(\mathrm{Em})]$, which may reflect porphyrin or protein-bound red-shifted flavin emission [46], is significantly affected by PGRMC1-HA phosphorylation status. The table provides Kolmogorov-Smirnov test $p$ values from pair wise comparisons. (B) The ratio of hyperspectral autofluorescence channels 3 [375 nm(Ex), $450 \mathrm{~nm}(E m)]$ to channel 12 [435 nm(Ex), $587 \mathrm{~nm}(E m)]$ differs significantly between cells. The table follows C. (C) Individual channels \#3 and \#4 from (B) as listed in Table S1 (left) and the same two channels superimposed (right).

Additional file 2 Fig. S2. PGRMC1 phosphorylation mutants do not affect AG-205-induced death. Related to Fig. 2. (A) AG-205-induced cell death is unaffected by PGRMC1 phosphorylation status. Cells were incubated in the presence of the indicated AG-205 concentrations ( $n=8: 3 x$ line 1, $3 x$ line 2, $2 x$ line 3) or DMSO vehicle control $(n=3: 1 x$ each cell line), and percentage viable cells was calculated relative to untreated cell controls ( $n=9: 3$ replicates per cell line) using MTT assay. (B) AUC results for values from A greater than $25 \mu \mathrm{M}$ reveal no significant differences in response to AG-205 treatment between cell lines ( $p>0.85$, post-hoc Bonferroni after 1 way ANOVA for AG-205 treatment). The apparently greater survival of DM cells at 25 MM AG-205 observed in this panel was never observed in multiple other repeat experiments.

Additional file $\mathbf{3}$ Fig. S3. SWATH-MS proteomic quantification of Nicotinamide phosphoribosyltransferase. Related to Fig. 6. The figure shows the abundance profile of P43490 nicotinamide phosphoribosyltransferase (NAMPT) from the SWATH-MS proteomics quantification of the accompanying manuscript [10].

Additional file 4 Fig. S4. PGRMC1 phosphorylation status affects the NNMT pathway. Related to Fig. 6. (A) Metabolomics quantification of Sadenosyl-Methionine (SAM) levels for the indicated cell lines, representing $n=12$ (four technical replicates for each of three independent cell lines per PGRMC1 condition). (B) RT-PCR quantification of NNMT mRNA levels 
after treatment by an NNMT-specific shRNA (NN) or a random scramble shRNA control (scr) in TM cells stably expressing lentiplasmid-driven shRNAs. RT-PCR Methods follow A. $p<0.003$ (2-tailed T test). Methods follow Fig. 6b. (C) 1-MNA ion intensities in cells from $B$, determined following methods from Fig. 6a, using two technical replicates of each of three biological replicates per cell condition $(n=6)$. Labels follow $\mathrm{B}$. The result was significantly different by T-test $(p<0.001)$ after removal of one Scr outlier (panel displayed) or by Mann-Whitney $U$ test $(p<0.002)$ including the outlier. (D) SAM ion intensities in the cells from B were not significantly different (T-test). Labels follow B. (E) 1-MNA attenuates rounded morphology in DM cells. Results for $n=5$ for each 1-MNA concentration are shown in the boxplot. $0.5 \mathrm{mM} 1$-MNA was significantly different $(p<$ 0.0001, ANOVA, post hoc Tukey HSD) to all other treatments.

Additional file $\mathbf{5}$ Fig. S5. Methylation status of $\mathrm{CpG}$ associated with annotated coding genes. Related to Fig. 7. Significant differentially methylated probes were annotated with the UCSC gene feature and the proportion of probes attributed to each feature counted. The $y$-axis shows the frequency of hypermethylated-hypomethylated probes per feature, and the $x$-axis shows the difference in those frequencies between WT/MP, DM/WT and TM/DM. 3'UTR: 3' untranslated region; 5'UTR: 5' untranslated region; Body: Between the ATG and stop codon; irrespective of the presence of introns, exons, TSS, or promoters; ExonBnd: exon boundaries: TSS1500/TSS200: within indicated number of residues of transcription start site (TSS).

Additional file 6 Fig. S6. Methylation status of $\mathrm{CpG}$ associated with annotated enhancers. Related to Fig. 7. Significant differentially methylated probes were annotated with the 450k_Enhancer feature and the proportion of probes attributed to each feature counted. Arrows indicate increased hypomethylation and reduced hypermethylation of enhancers in the TM/DM comparison.

Additional file $\mathbf{7}$ Fig. S7. Chromosomal context of differentially methylated $\mathrm{CpG}$, and biological association of genes. Related to Fig. 7. (A) CpG feature classifications for differentially methylated probes. The percentage of all significant probes that were hypermethylated or hypomethylated per CpG feature is presented for each comparison. Up arrows point to relatively greater fractions of Island and Shore genomic regions for the TM/DM comparison. The down arrow points to reduced OpenSea hypomethylation in the TM/DM comparison. (B) Intersections of significant KEGG pathways between WT/MP, DM/WT and TM/DM. KEGG enrichment was applied separately to significant hypermethylated and hypomethylated probes. The main bar chart presents the number of pathways common to each comparison. Each bar represents an intersect notated by linked dots on the $x$-axis. The number of significant pathways per comparison is presented as a horizontal bar chart. The KEGG results are in File S1 and the list of pathways unique to each comparison are provided. Pathways analysis of probes genes corresponding to the top 2000 most variable differentially methylated probes in CpG Island or Shore chromosomal regions. Probes were separated into hypermethylated and hypomethylated data sets from $\mathrm{D}$, including only probes from Island or Shores. Enriched Gene Ontology (GO) pathways enrichments are shown. The identities of pathways uniquely differential to one of the three cell type comparisons (WT/MP, DM/WT, or TM/DM) for both hypoand hyper-methylated data sets and KEGG pathways are available in Supplemental Information File 1.

Additional file 8 Table S1. Spectral channel filters employed for hyperspectral autofluorescence imaging. Related to Fig. 1. For further details of this approach see Gosnell et al. [44].

Additional file 9 Table S2. GO pathways enrichment results. Related to Fig. 7. The top ten $\mathrm{GO}$ enrichments for each cell comparison from Fig. 7. Full results are available in File S1.

Additional file $\mathbf{1 0}$ Table S3. Unique KEGG pathways detected for TM/ DM hyper and hypo-methylated gene sets. Related to Fig. 7. The identities of the 11 hypermethylated and 7 hypomethylated pathways unique to the TM/DM comparison from Fig. S7B are given. Full results are available in File $\mathrm{S} 1$.

Additional file 11 File S1. A zip archive containing excel files with the most significant pathways enrichment results from methylomics analysis including those of Fig. S7B, Table S2 and Table S3. The archive unpacks as five separate folders, each containing the following pathways analysis results. A) All GO enrichments for each cell comparison significant below the $\operatorname{adjP}=0.001$ level. B) All GO enrichments that were uique to a particular cell comparison. C) KEGG enriched pathways for each cell comparison, with analyses performed separately on hypermethyalted (files labelled up) and hyperemethylated (files labelled down) probes. These correspond to the pathways of of Fig. S7B. D) All KEGG enriched pathways from $C$ which were unique to a particular cell comparison. E) All All Reactome enriched pathways for each cell comparison significant below the adjp $=0.05$ level.

\section{Abbreviations}

1-MNA: 1-methylnicotinamide; adjP: Benjamini-Hochberg adjusted p-value; AG-205: AG-205 PGRMC1 inhibitor; CK2: Casein kinase 2; cyP450: cytochrome P450; CYP51A: Lanosterol 14-alpha demethylase; cytb5: cytochrome b5; Dap1: Damage-associated protein 1; DM: PGRMC1-HA S57A/S181A double mutant; Dox: Doxorubicin; GLP-1: Glucagon-like peptide-1; GO: Gene Ontology; GSK3- $\beta$ : Glycogen synthase kinase 3- $\beta$; HA: Hemagglutinin; hESCs: Human embryonic stem cells; KEGG: Kyoto Encyclopedia of Genes and Genomes; LC/MS: Liquid chromatography/mass spectrometry; LDL: Low density lipoprotein; LDLR: Low density lipoprotein receptor; MAPR: Membrane-associated progesterone receptor; MP: MIA PaCa-2 cells; mPRa: membrane progestin receptor a; NAMPT: Nicotinamide phosphoribosyltransferase; NNMT: Nicotinamide-N-methyltransferase; NpFR1: Naphthalimide-flavin redox sensor 1; NpFR2: Naphthalimide-flavin redox sensor 2; P4: Progesterone; PGRMC1: Progesterone Receptor Membrane Components 1; PGRMC1-HA: Hemagglutinin-tagged PGRMC1; RPPA: Reverse phase protein array; SAM: S-adenosylmethionine; SH2: Src homology 2; SH3: Src homology 3; TCF/LEF: T-cell factor/lymphoid enhancer factor; TM: PGRMC1-HA S57A/Y180F/S181A triple mutant; WT: PGRMC1-HA wild-type

\section{Acknowledgements}

The publication hiatus of more than a decade on this project has been caused by the Australian competitive grants system failing to support a single funding application from M.A.C. after his relocation with the PGRMC1 project from Germany to a regional Australian university. We thank Padraig Strappe for the gift of lentiviral helper packaging plasmids and HEK293 cells. MIA PaCa-2 (MP) pancreatic cancer cells were obtained from Dr. Patsy Soon, Kolling Institute of Medical Research, Sydney.

\section{Authors' contributions}

All authors have read and approved the manuscript. Conceptualization, M.A.C.; Methodology, M.A.C., P.P.A, B.M.T., E.M.G., M.G., E.J.N, L.A.W., P.A.W, and M.P; Formal Analysis, B.M.T., P.P.A., S.D.P., R.H.W., P.A.W., M.L., M.G., L.G., T.F. T.F., M.P. and M.A.C.; Investigation, B.M.T., P.P.A., S.L.T., J.F., P.A.W., L.A.W., S.G. and M.W.; Resources, M.P., E.J.N., H.N., T.F., E.M.G, and L.A.W.; Writing Original Draft, M.A.C., B.M.T.; Writing - Review \& Editing, M.A.C., B.M.T., P.P.A., M.P., E.M.G; Supervision, M.A.C, J.A.J., and E.J.N.; Project Administration, M.A.C.; Funding Acquisition, M.A.C., L.A.W., J.C.Q., E.M.G., H.N., T.F., and E.J.N.

\section{Funding}

This work has received no direct Australian competitive grant support since M.A.C.'s relocation to the country in 2008. The present results have been compiled largely due to the generosity of collaborating authors, and by the PhD stipends of B.M.T. and P.P.A. Research was primarily supported by Charles Sturt University (CSU) internal funds to M.A.C. J.A.J. and L.A.W. Open access publication fees were jointly supported by CSU's Faculty of Science, School of Biomedical Sciences, and Research Office. B.M.T. was supported by a PhD scholarship from the Ministry of Higher Education and Research, through the University of Wasit, Iraq. E.M.G. acknowledges partial support of Australian Research Council award CE140100003. E.J.N. acknowledges the support of the Australian Research Council (DE120102687) and the Ramaciotti Foundation (ES2012/0051).

\section{Availability of data and materials}

Methylomics data have been deposited in the ArrayExpress database at EMBL-EBI (www.ebi.ac.uk/arrayexpress) under accession number E-MTAB8218. Genomic sequencing results are available as NCBI Bioproject Accession: PRJNA400337, SRA Run Selector SRP118430 (https://www.ncbi.nlm.nih.gov/ 
bioproject/PRJNA400337). Further data are available in a supplemental information file available from the journal web page.

\section{Ethics approval and consent to participate}

Not applicable.

\section{Consent for publication}

Not applicable.

\section{Competing interests}

M.A.C. is scientific advisor to and minor shareholder of Cognition Therapeutics, a company developing sigma-2 receptor ligands against Alzheimer's disease. This work was performed independently of and without input from the company. The authors declare that they have no other competing interests.

\section{Author details}

${ }^{1}$ School of Biomedical Sciences, Charles Sturt University, Wagga Wagga, NSW 2650, Australia. ${ }^{2}$ Department of Biology, College of Science, University of Wasit, Kut, Wasit, Iraq. ${ }^{3}$ Present Address: Faculty of Pharmaceutical Sciences, The University of British Columbia, Vancouver, Canada. ${ }^{4}$ Graham Centre for Agricultural Innovation, Charles Sturt University, Boorooma Street, Wagga Wagga, NSW 2678, Australia. ${ }^{5}$ School of Agricultural and Wine Sciences, Charles Sturt University, Boorooma Street, Wagga Wagga, NSW 2678, Australia. ${ }^{6}$ ARC Centre of Excellence for Nanoscale BioPhotonics, Macquarie University, Sydney, NSW 2109, Australia. ${ }^{7}$ Present Address: The Graduate School of Biomedical Engineering, University of New South Wales, Sydney, Kensington, NSW 2052, Australia. ${ }^{8}$ Quantitative (Biotechnology) Pty. Ltd., ABN 17165684 186, Australiahttp://www.quantitative.net.au. ${ }^{9}$ Department of Gynecology and Obstetrics, University Women's Hospital of Dusseldorf, Dusseldorf, Germany. ${ }^{10}$ Australian Genome Research Facility Ltd., Victorian Comprehensive Cancer Centre, Melbourne, VIC 3000, Australia. ${ }^{11} \mathrm{NMI}$ TT Pharmaservices, Protein Profiling, 72770 Reutlingen, Germany. ${ }^{12}$ School of Animal and Veterinary Sciences, Charles Sturt University, Boorooma Street, Wagga Wagga, NSW 2678, Australia. ${ }^{13}$ Kinghorn Centre for Clinical Genomics, Garvan Institute of Medical Research, Darlinghurst, NSW 2010, Australia.

${ }^{14}$ ACRF Department of Cancer Biology and Therapeutics, The John Curtin School of Medical Research, The Australian National University, Canberra, ACT 2601, Australia. ${ }^{15}$ University of Sydney, School of Chemistry, Sydney, NSW 2006, Australia. ${ }^{16}$ Faculty of Science, Charles Sturt University, Boorooma Street, Wagga Wagga, NSW 2678, Australia.

\section{Received: 18 December 2019 Accepted: 20 March 2020} Published online: 15 April 2020

\section{References}

1. Ryu CS, Klein K, Zanger UM. Membrane associated progesterone receptors: promiscuous proteins with pleiotropic functions - focus on interactions with cytochromes P450. Front Pharmacol. 2017;8:159.

2. Cahill MA, Jazayeri JA, Catalano SM, Toyokuni S, Kovacevic Z, Richardson DR. The emerging role of progesterone receptor membrane component 1 (PGRMC1) in cancer biology. Biochim Biophys Acta. 2016; 1866(2):339-49.

3. Peluso JJ, Pru JK. Non-canonical progesterone signaling in granulosa cell function. Reproduction (Cambridge, England). 2014;147(5):R169-78.

4. Petersen SL, Intlekofer KA, Moura-Conlon PJ, Brewer DN, Del Pino SJ, Lopez JA. Nonclassical progesterone signalling molecules in the nervous system. J Neuroendocrinol. 2013;25(11):991-1001.

5. Cahill MA, Jazayeri JA, Kovacevic Z, Richardson DR. PGRMC1 regulation by phosphorylation: potential new insights in controlling biological activity. Oncotarget. 2016;7(32):50822-7.

6. Cahill MA. Progesterone receptor membrane component 1: an integrative review. J Steroid Biochem Mol Biol. 2007;105(1-5):16-36.

7. Ahmed IS, Rohe HJ, Twist KE, Mattingly MN, Craven RJ. Progesterone receptor membrane component 1 (Pgrmc1): a heme-1 domain protein that promotes tumorigenesis and is inhibited by a small molecule. J Pharmacol Exp Ther. 2010:333(2):564-73.

8. Hand RA, Craven RJ. Hpr6.6 protein mediates cell death from oxidative damage in MCF-7 human breast cancer cells. J Cell Biochem. 2003:90(3): 534-47.
9. Neubauer H, Clare SE, Wozny W, Schwall GP, Poznanovic S, Stegmann W, Vogel U, Sotlar K, Wallwiener D, Kurek R, et al. Breast cancer proteomics reveals correlation between estrogen receptor status and differential phosphorylation of PGRMC1. Breast Cancer Res. 2008;10(5):R85.

10. Thejer BM, Adhikary PP, Kaur A, Teakel SL, Van Oosterum A, Seth I, Pajic M Hannan KM, Pavy M, Poh P, et al. PGRMC1 phosphorylation affects cell shape, motility, glycolysis, mitochondrial form and function, and tumor growth. BMC Mol Cell Biol. 2020. https://doi.org/10.1186/s12860-1202000256-12863.

11. Kabe Y, Nakane T, Koike I, Yamamoto T, Sugiura Y, Harada E, Sugase K, Shimamura T, Ohmura M, Muraoka K, et al. Haem-dependent dimerization of PGRMC1/Sigma-2 receptor facilitates cancer proliferation and chemoresistance. Nat Commun. 2016:7:11030.

12. Hehenberger E, Eitel M, Fortunato SAV, Miller DJ, Keeling PJ, Cahill MA. Early eukaryotic origins and metazoan elaboration of MAPR family proteins. Mol Phylogenet Evol. https://doi.org/10.1016/j.ympev.2020.106814.

13. Piel RB 3rd, Shiferaw MT, Vashisht AA, Marcero JR, Praissman JL, Phillips JD, Wohlschlegel JA, Medlock AE. A novel role for progesterone receptor membrane component 1 (PGRMC1): a partner and regulator of Ferrochelatase. Biochemistry. 2016;55(37):5204-17.

14. Szczesna-Skorupa E, Kemper B. Progesterone receptor membrane component 1 inhibits the activity of drug-metabolizing cytochromes P450 and binds to cytochrome P450 reductase. Mol Pharmacol. 2011;79(3):340-50.

15. Oda S, Nakajima M, Toyoda Y, Fukami T, Yokoi T. Progesterone receptor membrane component 1 modulates human cytochrome p450 activities in an isoform-dependent manner. Drug Metab Dispos. 2011;39(11):2057-65.

16. Cahill MA, Medlock AE. Thoughts on interactions between PGRMC1 and diverse attested and potential hydrophobic ligands. J Steroid Biochem Mol Biol. 2017:171:11-33.

17. Hughes AL, Powell DW, Bard M, Eckstein J, Barbuch R, Link AJ, Espenshade PJ. Dap1/PGRMC1 binds and regulates cytochrome P450 enzymes. Cell Metab. 2007:5(2):143-9.

18. Mallory JC, Crudden G, Johnson BL, Mo C, Pierson CA, Bard M, Craven RJ. Dap1p, a heme-binding protein that regulates the cytochrome $\mathrm{P} 450$ protein Erg11p/Cyp51p in Saccharomyces cerevisiae. Mol Cell Biol. 2005;25(5):1669-79.

19. Mifsud W, Bateman A. Membrane-bound progesterone receptors contain a cytochrome b5-like ligand-binding domain. Genome Biol. 2002;3(12): RESEARCH0068.

20. Peluso JJ, Griffin D, Liu X, Horne M. Progesterone receptor membrane component1 (PGRMC1) and PGRMC-2 interact to suppress entry into the cell cycle in spontaneously immortalized rat granulosa cells. Biol Reprod. 2014;91(5):104.

21. Griffin D, Liu X, Pru C, Pru JK, Peluso JJ. Expression of progesterone receptor membrane component- 2 within the immature rat ovary and its role in regulating mitosis and apoptosis of spontaneously immortalized granulosa cells. Biol Reprod. 2014;91(2):36.

22. Juhlen R, Landgraf D, Huebner A, Koehler K. Triple a patient cells suffering from mitotic defects fail to localize PGRMC1 to mitotic kinetochore fibers. Cell Div. 2018;13:8.

23. Luciano AM, Peluso JJ. PGRMC1 and the faithful progression through mitosis and meiosis. Cell Cycle. 2016;15(17):2239-40.

24. Terzaghi L, Tessaro I, Raucci F, Merico V, Mazzini G, Garagna S, Zuccotti M, Franciosi F, Lodde V. PGRMC1 participates in late events of bovine granulosa cells mitosis and oocyte meiosis. Cell Cycle. 2016;15(15):2019-32.

25. Sakamoto $\mathrm{H}$, Ukena K, Takemori H, Okamoto M, Kawata M, Tsutsui K. Expression and localization of 25-dx, a membrane-associated putative progesterone-binding protein, in the developing Purkinje cell. Neuroscience. 2004;126(2):325-34

26. Izzo NJ, Xu J, Zeng C, Kirk MJ, Mozzoni K, Silky C, Rehak C, Yurko R, Look G, Rishton $\mathrm{G}$, et al. Alzheimer's therapeutics targeting amyloid beta 1-42 oligomers II: Sigma-2/PGRMC1 receptors mediate Abeta 42 oligomer binding and synaptotoxicity. PLoS One. 2014;9(11):e111899.

27. Kim JY, Kim SY, Choi HS, An S, Ryu CJ. Epitope mapping of anti-PGRMC1 antibodies reveals the non-conventional membrane topology of PGRMC1 on the cell surface. Sci Rep. 2019;9(1):653.

28. Terzaghi L, Luciano AM, Dall'Acqua PC, Modina SC, Peluso JJ, Lodde V, et al. Reproduction. 2018;155(3):273-82.

29. Galmozzi A, Kok BP, Kim AS, Montenegro-Burke JR, Lee JY, Spreafico R, Mosure S, Albert V, Cintron-Colon R, Godio C, et al. PGRMC2 is an intracellular haem chaperone critical for adipocyte function. Nature. 2019; 576(7785):138-42 
30. Rohe HJ, Ahmed IS, Twist KE, Craven RJ. PGRMC1 (progesterone receptor membrane component 1): a targetable protein with multiple functions in steroid signaling, P450 activation and drug binding. Pharmacol Ther. 2009;121(1):14-9.

31. Hand RA, Jia N, Bard M, Craven RJ. Saccharomyces cerevisiae Dap1p, a novel DNA damage response protein related to the mammalian membraneassociated progesterone receptor. Eukaryot Cell. 2003;2(2):306-17.

32. Suchanek M, Radzikowska A, Thiele C. Photo-leucine and photo-methionine allow identification of protein-protein interactions in living cells. Nat Methods. 2005;2(4):261-7.

33. Cahill MA. The evolutionary appearance of signaling motifs in PGRMC1. Bioscience Trends. 2017;11(2):179-92.

34. Riad A, Zeng C, Weng CC, Winters H, Xu K, Makvandi M, Metz T, Carlin S, Mach $\mathrm{RH}$. Sigma-2 receptor/TMEM97 and PGRMC-1 increase the rate of internalization of $L D L$ by $L D L$ receptor through the formation of a ternary complex. Sci Rep. 2018;8(1):16845.

35. Lin ST, May EW, Chang JF, Hu RY, Wang LH, Chan HL. PGRMC1 contributes to doxorubicin-induced chemoresistance in MES-SA uterine sarcoma. Cell Mol Life Sci. 2015;72(12):2395-409.

36. Friel AM, Zhang L, Pru CA, Clark NC, McCallum ML, Blok LJ, Shioda T, Peluso $J$ J, Rueda BR, Pru JK. Progesterone receptor membrane component 1 deficiency attenuates growth while promoting chemosensitivity of human endometrial xenograft tumors. Cancer Lett. 2015;356(2 Pt B):434-42.

37. Peluso JJ, Romak J, Liu X. Progesterone receptor membrane component-1 (PGRMC1) is the mediator of progesterone's antiapoptotic action in spontaneously immortalized granulosa cells as revealed by PGRMC1 small interfering ribonucleic acid treatment and functional analysis of PGRMC1 mutations. Endocrinology. 2008;149(2):534-43.

38. Kaczanowski S. Apoptosis: its origin, history, maintenance and the medical implications for cancer and aging. Phys Biol. 2016;13(3):031001.

39. Teakel SL, Ludescher M, Thejer BM, Poschmann G, Forwood JK, Neubauer H, Cahill MA. Protein complexes including PGRMC1 and actin-associated proteins are disrupted by AG-205. Biochemical and biophysical research communications 2020, In Press. Accepted 20 December, 2019. Published online 21 January; 2020. https://doi.org/10.1016/j.bbrc.2019.1012.1108.

40. Yoshitani N, Satou K, Saito K, Suzuki S, Hatanaka H, Seki M, Shinozaki K, Hirota $\mathrm{H}$, Yokoyama S. A structure-based strategy for discovery of small ligands binding to functionally unknown proteins: combination of in silico screening and surface plasmon resonance measurements. Proteomics. 2005:5(6):1472-80.

41. Elliott AD, Gao L, Ustione A, Bedard N, Kester R, Piston DW, Tkaczyk TS. Realtime hyperspectral fluorescence imaging of pancreatic beta-cell dynamics with the image mapping spectrometer. J Cell Sci. 2012;125(Pt 20):4833-40

42. Lu G, Fei B. Medical hyperspectral imaging: a review. J Biomed Opt. 2014; 19(1):10901.

43. Gosnell ME, Anwer AG, Mahbub SB, Menon Perinchery S, Inglis DW, Adhikary PP, Jazayeri JA, Cahill MA, Saad S, Pollock CA, et al. Quantitative non-invasive cell characterisation and discrimination based on multispectral autofluorescence features. Sci Rep. 2016;6:23453.

44. Gosnell ME, Anwer AG, Cassano JC, Sue CM, Goldys EM. Functional hyperspectral imaging captures subtle details of cell metabolism in olfactory neurosphere cells, disease-specific models of neurodegenerative disorders. Biochim Biophys Acta. 2016;1863(1):56-63.

45. Huang S, Heikal AA, Webb WW. Two-photon fluorescence spectroscopy and microscopy of NAD(P)H and flavoprotein. Biophys J. 2002;82(5):2811-25.

46. Schneckenburger $H$, Lang M, Köllner T, Rück A, Herzog M, Hörauf H, Steiner $R$. Fluorescence spectra and microscopic imaging of porphyrins in single cells and tissues. Laser Med Sci. 1989;4(3):159-66.

47. Clark NC, Friel AM, Pru CA, Zhang L, Shioda T, Rueda BR, Peluso JJ, Pru JK. Progesterone receptor membrane component 1 promotes survival of human breast cancer cells and the growth of xenograft tumors. Cancer Biol Ther. 2016;17(3):262-71.

48. Kaur A, Brigden KW, Cashman TF, Fraser ST, New EJ. Mitochondrially targeted redox probe reveals the variations in oxidative capacity of the haematopoietic cells. Organ Biomol Chem. 2015;13(24):6686-9.

49. Yeow J, Kaur A, Anscomb MD, New EJ. A novel flavin derivative reveals the impact of glucose on oxidative stress in adipocytes. Chem Commun (Camb). 2014;50(60):8181-4.

50. Canto C, Menzies KJ, Auwerx J. NAD(+) metabolism and the control of energy homeostasis: a balancing act between mitochondria and the nucleus. Cell Metab. 2015;22(1):31-53.

51. Schultz MB, Sinclair DA. Why NAD(+) declines during aging: It's destroyed. Cell Metab. 2016;23(6):965-6.
52. Sperber H, Mathieu J, Wang Y, Ferreccio A, Hesson J, Xu Z, Fischer KA, Devi A, Detraux D, Gu H, et al. The metabolome regulates the epigenetic landscape during naive-to-primed human embryonic stem cell transition. Nat Cell Biol. 2015;17(12):1523-35.

53. Hong S, Moreno-Navarrete JM, Wei X, Kikukawa Y, Tzameli I, Prasad D, Lee Y, Asara JM, Fernandez-Real JM, Maratos-Flier E, et al. Nicotinamide Nmethyltransferase regulates hepatic nutrient metabolism through Sirt1 protein stabilization. Nat Med. 2015;21(8):887-94.

54. Jones PA. Functions of DNA methylation: islands, start sites, gene bodies and beyond. Nat Rev Genet. 2012;13(7):484-92.

55. Kiefer JC. Epigenetics in development. Dev Dyn. 2007;236(4):1144-56.

56. Nishino K, Umezawa A. DNA methylation dynamics in human induced pluripotent stem cells. Hum Cell. 2016;29(3):97-100.

57. Kim JY, Kim SY, Choi HS, Kim MK, Lee HM, Jang YJ, Ryu CJ. Progesterone receptor membrane component 1 suppresses the p53 and Wnt/betacatenin pathways to promote human pluripotent stem cell self-renewal. Sci Rep. 2018;8(1):3048.

58. Cadigan KM, Waterman ML. TCF/LEFs and Wnt signaling in the nucleus. Cold Spring Harb Perspect Biol. 2012;4(11).

59. Kraus Y, Aman A, Technau U, Genikhovich G. Pre-bilaterian origin of the blastoporal axial organizer. Nat Commun. 2016;7:11694.

60. Nielsen C, Brunet T, Arendt D. Evolution of the bilaterian mouth and anus. Nat Ecol Evol. 2018;2(9):1358-76.

61. Genikhovich G, Technau U. On the evolution of bilaterality. Development. 2017;144(19):3392-404

62. Hermida MA, Dinesh Kumar J, Leslie NR. GSK3 and its interactions with the PI3KJAKT/mTOR signalling network. Adv Biol Regul. 2017;65:5-15.

63. Peluso JJ, DeCerbo J, Lodde V. Evidence for a genomic mechanism of action for progesterone receptor membrane component-1. Steroids. 2012; 77(10):1007-12

64. Petralia RS, Mattson MP, Yao PJ. Aging and longevity in the simplest animals and the quest for immortality. Ageing Res Rev. 2014;16:66-82.

65. Shih CC, Chou HC, Chen YJ, Kuo WH, Chan CH, Lin YC, Liao EC, Chang SJ, Chan HL. Role of PGRMC1 in cell physiology of cervical cancer. Life Sci. 2019;231(19):116541

66. Schmeisser K, Parker JA. Nicotinamide-N-methyltransferase controls behavior, neurodegeneration and lifespan by regulating neuronal autophagy. PLoS Genet. 2018;14(9):e1007561.

67. Neelakantan H, Brightwell CR, Graber TG, Maroto R, Wang HL, McHardy SF, Papaconstantinou J, Fry CS, Watowich SJ. Small molecule nicotinamide Nmethyltransferase inhibitor activates senescent muscle stem cells and improves regenerative capacity of aged skeletal muscle. Biochem Pharmacol. 2019;163:481-92.

68. Schmeisser K, Mansfeld J, Kuhlow D, Weimer S, Priebe S, Heiland I, Birringer M, Groth M, Segref A, Kanfi $Y$, et al. Role of sirtuins in lifespan regulation is linked to methylation of nicotinamide. Nat Chem Biol. 2013;9(11):693-700.

69. Calvani R, Brasili E, Pratico G, Capuani G, Tomassini A, Marini F, Sciubba F, Finamore A, Roselli M, Marzetti E, et al. Fecal and urinary NMR-based metabolomics unveil an aging signature in mice. Exp Gerontol. 2014;49: $5-11$.

70. Kraus D, Yang Q, Kong D, Banks AS, Zhang L, Rodgers JT, Pirinen E, Pulinilkunnil TC, Gong F, Wang YC, et al. Nicotinamide N-methyltransferase knockdown protects against diet-induced obesity. Nature. 2014;508(7495): 258-62.

71. Strom K, Morales-Alamo D, Ottosson F, Edlund A, Hjort L, Jorgensen SW, Almgren P, Zhou Y, Martin-Rincon M, Ekman C, et al. N(1)methylnicotinamide is a signalling molecule produced in skeletal muscle coordinating energy metabolism. Sci Rep. 2018;8(1):3016.

72. Jung J, Kim LJ, Wang X, Wu Q, Sanvoranart T, Hubert CG, Prager BC, Wallace LC, Jin X, Mack SC, et al. Nicotinamide metabolism regulates glioblastoma stem cell maintenance. JCI Insight. 2017:2(10):90019.

73. Xie X, Yu H, Wang Y, Zhou Y, Li G, Ruan Z, Li F, Wang X, Liu H, Zhang J. Nicotinamide $\mathrm{N}$-methyltransferase enhances the capacity of tumorigenesis associated with the promotion of cell cycle progression in human colorectal cancer cells. Arch Biochem Biophys. 2014;564:52-66.

74. Ulanovskaya OA, Zuhl AM, Cravatt BF. NNMT promotes epigenetic remodeling in cancer by creating a metabolic methylation sink. Nat Chem Biol. 2013;9(5):300-6.

75. Pissios P. Nicotinamide $\mathrm{N}$-methyltransferase: more than a vitamin B3 clearance enzyme. Trends Endocrinol Metab. 2017;28(5):340-53. 
76. Revollo JR, Grimm AA, Imai S. The NAD biosynthesis pathway mediated by nicotinamide phosphoribosyltransferase regulates Sir2 activity in mammalian cells. J Biol Chem. 2004;279(49):50754-63.

77. Dai H, Sinclair DA, Ellis JL, Steegborn C. Sirtuin activators and inhibitors: promises, achievements, and challenges. Pharmacol Ther. 2018;188:140-54.

78. Wrigley S, Arafa D, Tropea D. Insulin-like growth factor 1: at the crossroads of brain development and aging. Front Cell Neurosci. 2017;11:14.

79. Franchin C, Borgo C, Cesaro L, Zaramella S, Vilardell J, Salvi M, Arrigoni G, Pinna LA. Re-evaluation of protein kinase CK2 pleiotropy: new insights provided by a phosphoproteomics analysis of CK2 knockout cells. Cell Mol Life Sci. 2018;75(11):2011-26.

80. Willibald M, Bayer G, Stahlhut V, Poschmann G, Stuhler K, Gierke B, Pawlak $M$, Seeger $H$, Mueck AO, Niederacher $D$, et al. Progesterone receptor membrane component 1 is phosphorylated upon progestin treatment in breast cancer cells. Oncotarget. 2017;8(42):72480-93.

81. Willibald M, Wurster I, Meisner C, Vogel U, Seeger H, Mueck AO, Fehm T, Neubauer H. High Level of Progesteron Receptor Membrane Component 1 (PGRMC 1) in Tissue of Breast Cancer Patients is Associated with Worse Response to Anthracycline-Based Neoadjuvant Therapy. Horm Metab Res. 2017;49(8):595-603.

82. Neubauer H, Adam G, Seeger H, Mueck AO, Solomayer E, Wallwiener D, Cahill MA, Fehm T. Membrane-initiated effects of progesterone on proliferation and activation of VEGF in breast cancer cells. Climacteric. 2009; 12(3):230-9.

83. Pagano MA, Cesaro L, Meggio F, Pinna LA. Protein kinase CK2: a newcomer in the 'druggable kinome'. Biochem Soc Trans. 2006;34(Pt 6):1303-6.

84. Parsons RB, Aravindan S, Kadampeswaran A, Evans EA, Sandhu KK, Levy ER, Thomas MG, Austen BM, Ramsden DB. The expression of nicotinamide Nmethyltransferase increases ATP synthesis and protects SH-SY5Y neuroblastoma cells against the toxicity of complex I inhibitors. Biochem J. 2011;436(1):145-55.

85. Huber W, Carey VJ, Gentleman R, Anders S, Carlson M, Carvalho BS, Bravo HC, Davis S, Gatto L, Girke T, et al. Orchestrating high-throughput genomic analysis with bioconductor. Nat Methods. 2015;12(2):115-21.

86. Ritchie ME, Phipson B, Wu D, Hu Y, Law CW, Shi W, Smyth GK. limma powers differential expression analyses for RNA-sequencing and microarray studies. Nucleic Acids Res. 2015:43(7):e47.

87. Yu G, He QY, ReactomePA. an R/bioconductor package for reactome pathway analysis and visualization. Mol BioSyst. 2016;12(2):477-9.

88. Falcon S, Gentleman R. Using GOstats to test gene lists for GO term association. Bioinformatics. 2007;23(2):257-8.

89. Conway JR, Lex A, Gehlenborg N. UpSetR: an R package for the visualization of intersecting sets and their properties. Bioinformatics. 2017;33(18):2938-40.

\section{Publisher's Note}

Springer Nature remains neutral with regard to jurisdictional claims in published maps and institutional affiliations.

Ready to submit your research? Choose BMC and benefit from:

- fast, convenient online submission

- thorough peer review by experienced researchers in your field

- rapid publication on acceptance

- support for research data, including large and complex data types

- gold Open Access which fosters wider collaboration and increased citations

- maximum visibility for your research: over $100 \mathrm{M}$ website views per year

At BMC, research is always in progress.

Learn more biomedcentral.com/submissions 